COMMUNICATIONS IN

ANALYSIS AND GEOMETRY

Volume 12, Number 4, 887-926, 2004

\title{
Painlevé Expansions, Cohomogeneity One Metrics and Exceptional Holonomy
}

\author{
Andrew Dancer And McKenzIE Y. WAng
}

\section{Introduction.}

In this paper we continue our study ([DW1] - [DW4]) of ordinary differential equations arising as reductions of the Einstein equations. One way of performing this reduction is to require the Einstein metrics to be of cohomogeneity one-that is, they are required to be invariant under the action of a group with principal orbits of codimension one.

In [DW4] we introduced variables so that the cohomogeneity one Ricciflat equations became a constrained flow of an ODE system with quadratic nonlinearities. This enabled us to perform a Painlevé-Kowalewski analysis (cf. $[\mathrm{ARS}],[\mathrm{AvM}]$ ) of these equations in the case when the isotropy representation had two inequivalent summands. In Painlevé-Kowalewski analysis, one looks for singular solutions of a system of ODEs (containing several parameters) given by Painlevé expansions, i.e., meromorphic expansions in (a rational power of) the independent variable. The singularity (poles or branch points) is movable (i.e., its position can be continuously varied), and should be distinguished (in the particular case of the Einstein equations) from singularities of the metric. The general philosophy of this method is that the existence of large families of Painlevé expansions should be associated with "nice" properties of the equations, and in general occurs for only special values of the parameters in the equations. In particular, if for each dependent variable in the equations there is a corresponding family of Painlevé expansions which depends on the maximum number of degrees of freedom and in which that dependent variable actually blows up, then this is regarded as a strong indication that the equations are "integrable".

In this paper we shall prove some general results about the Painlevé analysis of the cohomogeneity 1 Ricci-flat equations in the situation when the isotropy representation of the principal orbit consists of pairwise inequivalent

\footnotetext{
${ }^{1}$ Mathematics Subject Classification (2000): 53C25, 53C29, 34E05.
} 
representations. We then apply these results to examples of more complicated orbit types than those in [DW4], including some which have recently become relevant in the study of metrics of exceptional holonomy in string theory ([CGLP1], [BGGG]).

As in [DW4], we find that existence of nontrivial Painlevé expansions depends sensitively on the choice of principal orbit, and is often associated with the existence of a solution to a Diophantine problem (in particular, the existence of an integral point on an elliptic curve) ( $\operatorname{cf} \S 6$ and $\S 7)$. We also find that, when Painlevé expansions do exist, they sometimes represent metrics of exceptional holonomy. Indeed, for some families of principal orbits (cf $\S 6$ ) the Diophantine constraints appear to single out precisely the dimensions in which exceptional holonomy metrics can live.

The paper is organised as follows. In $\S 2$ we perform a general analysis of the possible leading terms of a Painlevé expansion for our Ricci-flat equations. We also relate the leading term behaviour of our Painlevé expansions to the end behaviour of the corresponding Ricci-flat metrics. In $\S 3$ we study the resonances, that is, the steps in the expansion at which a free parameter may enter. These are the steps at which the linear operator in the recursion relation for the series expansion is noninvertible. Existence of a nontrivial Painlevé expansion requires the existence of rational resonances, giving rise to the Diophantine conditions mentioned above. In $\S 4$ we discuss compatibility conditions for the recursion to proceed at each resonance, as well as the constraints that the ODE flow satisfies. In $\S 5$ and $\S 6$ we apply this general theory to some cases of particular interest including ones where metrics of exceptional holonomy have recently been shown to exist. We list the possible Painlevé expansions, find the number of free parameters in each, and discuss their geometric significance. In particular, we discuss the Painlevé expansions which represent solutions of the equations for $G_{2}$ and $\operatorname{Spin}(7)$ holonomy. Finally, $\S 7$ contains a discussion of the Diophantine equations which arise from the requirement of rationality of resonances.

In the sequel [DW6] to this paper, the techniques in $\S 2-\S 4$ will be applied further to the Ricci-flat systems where the principal orbits are the Aloff-Wallach spaces and certain principal circle bundles over a product of two Fano varieties.

Acknowledgements The first author wishes to thank the Clay Mathematics Institute and the Isaac Newton Institute for their warm hospitality during the Geometry and String Theory School in March/April 2002. The second author is partially supported by NSERC grant OPG0009421. We are grateful to Gary Gibbons and Chris Pope for discussions on cohomogeneity 
one metrics. Finally, we thank John Wilson and Adam Logan for help with Diophantine equations, and Emmanuel Hermann for answering questions about the algorithms in SIMATH.

\section{The equations.}

In this section we briefly review the formulation in [DW4] of the cohomogeneity one Ricci-flat equations (see also [BB], [DW1], [EW] for background on these equations).

Consider a Riemannian manifold $(\mathcal{M}, g)$ with a cohomogeneity one isometric action of a compact Lie group $G$, whose principal orbit is $G / K$. We denote by

$$
\mathfrak{p}=\mathfrak{p}_{1} \oplus \ldots \oplus \mathfrak{p}_{r}
$$

the decomposition of the isotropy representation $\mathfrak{p} \approx T_{(K)}(G / K)$ of $G / K$ into irreducible $K$-representations. We let $d_{i}$ be the real dimension of $\mathfrak{p}_{i}$, and let $n=\sum_{i=1}^{r} d_{i}$ denote the dimension of $G / K(\operatorname{so} \operatorname{dim} \mathcal{M}=n+1)$. We use $d$ for the vector of dimensions $\left(d_{1}, \ldots, d_{r}\right)$.

We shall assume that the isotropy representation is monotypic, that is, all the $\mathfrak{p}_{i}$ are distinct as representations of $K$. In particular if there is a trivial summand it must be 1-dimensional. The metric $g$ may now be written as

$$
d t^{2}+g_{t}
$$

where

$$
g_{t}=\left.\left.e^{q_{1}(t)} B\right|_{\mathfrak{p}_{1}} \perp \ldots \perp e^{q_{r}(t)} B\right|_{\mathfrak{p}_{r}}
$$

is a 1-parameter family of $G$-invariant metrics on $G / K, B$ is a background metric on $G / K$ induced from some biinvariant metric on $G$, and $t$ is a parameter along a geodesic which intersects all principal orbits orthogonally. Note that the factor $\exp \left(\frac{1}{2} d \cdot q\right)$ is the ratio of the volume of $g_{t}$ to that of $B$.

The scalar curvature of $\left(G / K, g_{t}\right)$ is given by

$$
\mathrm{S}=\sum_{w \in \mathcal{W}} A_{w} e^{w \cdot q}
$$

where $A_{w}$ are (nonzero) constants and $\mathcal{W}$ is a finite collection of vectors $w \in \mathbb{Z}^{r}$ (which depend only on $G / K$ and which we refer to as weight vectors). These vectors fall into three types

(i) Type I: one entry of $w$ is -1 , the others are zero,

(ii) Type II: one entry is 1 , two are -1 , the rest are zero, 
(iii) Type III: one entry is 1 , one is -2 , the rest are zero.

We define a non-negative integer $\ell$ by

$$
r+\ell=|\mathcal{W}|
$$

We shall also assume that the elements of $\mathcal{W}$ span $\mathbb{R}^{r}$, which is the case when $G$ is semisimple (cf proof of Theorem 3.11 in [DW1]). So we may order the weight vectors as $w^{(k)}(1 \leq k \leq r+\ell)$, where

$$
w^{(i+r)}=\sum_{j=1}^{r} \nu_{i j} w^{(j)} \quad: \quad 1 \leq i \leq \ell
$$

for some constants $\nu_{i j}$. Note that as $w^{(k)} \cdot(1, \ldots, 1)=-1$, we can deduce that $\sum_{j=1}^{r} \nu_{i j}=1$ for all $i$. We shall refer to the constant $A_{w^{(k)}}$ just as $A_{k}$ in future.

As explained in [DW4], the Ricci-flat equations for $g$ may be viewed as the flow for a Hamiltonian $\bar{H}$ together with the constraint $\bar{H}=0$. The potential term is the scalar curvature $S$ of $G / K$ times the square of the volume distortion factor $\exp \left(\frac{1}{2} d \cdot q\right)$. The kinetic energy is given by the signature $(1, r-1)$ quadratic form

$$
J(p)=\frac{1}{n-1}\left(\sum_{i=1}^{r} p_{i}\right)^{2}-\sum_{i=1}^{r} \frac{p_{i}^{2}}{d_{i}} .
$$

In [DW4] we showed how to rewrite the Ricci-flat equations as a quadratic system, using ideas from $[\mathrm{AvM}]$.

We first define an $(r+\ell) \times r$ matrix $\hat{U}$ by

$$
\hat{U}_{i j}=d_{j}+w_{j}^{(i)} .
$$

Consider the matrix $\Phi:=\hat{U} J \hat{U}^{t}$, where we have also denoted by $J$ the matrix of the quadratic form given in (1.3). Now we can choose an $(r+\ell) \times r$ matrix $Q$ satisfying $Q D Q^{t}=\Phi$, where $D=\operatorname{diag}\left(\epsilon_{1}, \ldots, \epsilon_{r}\right)$ and

$$
\epsilon_{1}=1 \quad: \quad \epsilon_{i}=-1 \quad(i>1) .
$$

We may also arrange that $Q$ is of the form

$$
\left(\begin{array}{c}
Q_{1} \\
\nu Q_{1}
\end{array}\right)
$$


for some invertible $r \times r$ matrix $Q_{1}$.

The Ricci-flat equations for $g$ are now equivalent, after suitable change of dependent and independent variables, to the system

$$
\begin{aligned}
z_{i}^{\prime}=2 z_{i} \sum_{j=1}^{r} Q_{i j} v_{j}: & (1 \leq i \leq r+\ell), \\
v_{i}^{\prime} & =\epsilon_{i} \sum_{j=1}^{r+\ell} Q_{j i} z_{j}: \quad(1 \leq i \leq r),
\end{aligned}
$$

together with the constraints

$$
\frac{z_{r+j}}{A_{r+j}}=\prod_{i=1}^{r}\left(\frac{z_{i}}{A_{i}}\right)^{\nu_{j i}} \quad: \quad(1 \leq j \leq \ell)
$$

and the Hamiltonian constraint

$$
\bar{H} \equiv v_{1}^{2}-v_{2}^{2}-\ldots-v_{r}^{2}-\sum_{j=1}^{r+\ell} z_{j}=0 .
$$

The quantities $z_{r+j} \prod_{i=1}^{r} z_{i}^{-\nu_{j i}}$, as well as $\bar{H}$, are conserved by the flow. As discussed in [DW4], equations (1.5)-(1.6) may be viewed as a Poisson Hamiltonian flow, and the constraints (1.7) define restriction of this flow to a symplectic leaf. The Ricci-flat equations are then the Hamiltonian flow on this leaf subject to the Hamiltonian constraint (1.8).

Note that the choice of $Q$ above is not unique. Indeed, replacing $Q$ by $-Q$ and $v_{j}$ by $-v_{j}$ will leave the above system invariant.

\section{Leading terms.}

We can now begin the analysis of equations (1.5)-(1.6). The references $[\mathrm{ARS}]$ and $[\mathrm{AvM}]$ provide a good source for a modern treatment of PainlevéKowalewski methods. We should point out that one of the advantages of working with a quadratic system such as (1.5)-(1.6) is that once we establish the existence of a formal Painlevé expansion in a rational power of the independent variable, its convergence in a deleted neighbourhood of 0 follows from a general majorisation argument ( $\mathrm{cf} \S 6$ in [DW2]). 


\section{A. Algorithm for their determination}

We first look for possible leading terms of the Painlevé expansions. We denote the independent variable by $s$. Put

$$
z_{i}=\alpha_{0}^{(i)} s^{m_{i}}+\ldots, \quad: \quad v_{i}=\beta_{0}^{(i)} s^{n_{i}}+\ldots
$$

where $\alpha_{0}^{(i)}, \beta_{0}^{(i)} \neq 0$.

Let $n_{\min }$ be the least of the $n_{i}$ and suppose $n_{\min }<-1$, We see from (1.5) that for all $i$,

$$
\sum_{\left\{j: n_{j}=n_{\min }\right\}} Q_{i j} \beta_{0}^{(j)}=0,
$$

which is impossible because $Q_{1}$ and hence $Q$ have maximal rank. So all $n_{i}$ are $\geq-1$.

In the following we will deal with the important case where all $m_{i} \geq-2$. See, however, Remark 2.14 for a discussion of what happens if some $m_{i}<-2$.

Let $S \subset\{1, \ldots, r+\ell\}$ denote the set of indices $i$ for which $m_{i}=-2$ and $T \subset\{1, \ldots, r\}$ denote the set of indices for which $n_{i}=-1$. We will make the convention that $i \notin S$ means that $i$ belongs to the complement of $S$ in $\{1, \ldots, r+\ell\}$. Similarly, we say $j \notin T$ if $j$ belongs to the complement of $T$ in $\{1, \ldots, r\}$. Substituting (2.1) into (1.5)-(1.6) we obtain

$$
\begin{aligned}
m_{i} & =2 \sum_{j \in T} Q_{i j} \beta_{0}^{(j)}, \\
\beta_{0}^{(i)} & =-\epsilon_{i} \sum_{j \in S} Q_{j i} \alpha_{0}^{(j)} \quad \text { if } i \in T, \\
0 & =\sum_{j \in S} Q_{j i} \alpha_{0}^{(j)} \quad \text { if } i \notin T .
\end{aligned}
$$

(These equations are valid even if some of the $m_{i}, n_{i}$ are zero.)

It is convenient to introduce a $(r+\ell) \times r$ matrix $P$ and $r \times 1$ vector $\hat{\beta}_{0}$ defined by

$$
\begin{aligned}
& P_{i j}=Q_{i j} \quad \text { if } i \in S \\
& =0 \text { if } i \notin S \text {, } \\
& \hat{\beta}_{0}^{(i)}=\beta_{0}^{(i)} \quad \text { if } i \in T \\
& =0 \text { if } i \notin T \text {. }
\end{aligned}
$$

We can now rewrite equations (2.2)-(2.4) as

$$
\begin{aligned}
& m=2 Q \hat{\beta}_{0}, \\
& \hat{\beta}_{0}=-D P^{t} \alpha_{0},
\end{aligned}
$$


where $m$ is the column vector whose $i$ th entry is $m_{i}$ and $D=\operatorname{diag}\left(\epsilon_{1}, \ldots, \epsilon_{r}\right)$.

It follows that $m=-2 Q D P^{t} \alpha_{0}$, so

$$
m_{i}=-2 \sum_{j \in S}\left(Q D Q^{t}\right)_{i j} \alpha_{0}^{(j)}=-2 \sum_{j \in S}\left(\hat{U} J \hat{U}^{t}\right)_{i j} \alpha_{0}^{(j)} .
$$

Let $\Phi^{S}$ denote the $(r+\ell) \times|S|$ matrix obtained by deleting the $j$ th column of $\Phi=\hat{U} J \hat{U}^{t}$ iff $j \notin S$, and let $\hat{\alpha_{0}}$ denote the $|S| \times 1$ vector $\left(\alpha_{0}^{(i)}\right)_{i \in S}$. We now see

$$
m=-2 \Phi^{S} \hat{\alpha_{0}} .
$$

Therefore we need

$$
\begin{aligned}
& \left(\Phi^{S} \hat{\alpha_{0}}\right)^{(i)}=1 \quad(i \in S), \\
& \left(\Phi^{S} \hat{\alpha_{0}}\right)^{(i)}<1 \quad(i \notin S) .
\end{aligned}
$$

This gives us an algorithm to find the possibilities for $S, \alpha_{0}$ and $\beta_{0}$. For each subset $S$ of $\{1, \ldots, r+\ell\}$, we solve (2.8) for $\hat{\alpha_{0}}$ and then check if (2.9) is satisfied. (If $|S|<r+\ell$ then the components $\alpha_{0}^{(i)}$ with $i \notin S$ will be free.) Equation (2.6) now gives us the corresponding possibilities for $\beta_{0}$. If $|T|<r$ then some of the entries in $\hat{\beta}_{0}$ will be zero, and the corresponding entries of $\beta_{0}$ will be free.

Remark 2.10. Note that if $w^{(j)}$ lies in the span of $\left\{w^{(i)}: i \in S\right\}$ then, since $w^{(i)} \cdot(1, \ldots, 1)=-1$ for all $i$, we can write

$$
w^{(j)}=\sum_{i \in S} \sigma_{j i} w^{(i)} \quad \text { where } \sum_{i \in S} \sigma_{j i}=1,
$$

and hence

$$
d+w^{(j)}=\sum_{i \in S} \sigma_{j i}\left(d+w^{(i)}\right) .
$$

It now follows that for $\Phi$, and hence for $\Phi^{S}$,

$$
\text { row } j=\sum_{i \in S} \sigma_{j i} \text { row } i,
$$

so, from (2.7),

$$
m_{j}=\sum_{i \in S} \sigma_{j i} m_{i}=-2 \sum_{i \in S} \sigma_{j i}=-2,
$$

that is, $j \in S$. This discussion shows that we only have to apply the above algorithm to sets $S$ which satisfy the property

$$
j \in S \text { whenever } w^{(j)} \in \operatorname{Span}\left\{w^{(i)}: i \in S\right\} .
$$


Remark 2.12. If $S$ is a singleton $\{k\}$ our algorithm takes a particularly simple form. Now conditions (2.8)-(2.9) are satisfied if and only if either $\Phi_{k k}$ is positive and the greatest element in its column, or negative and the least element in its column.

Example 2.13. In our earlier paper [DW4] we considered the case of $r=2$ with weight vectors $(-1,0),(0,-1),(1,-2)$ (so $\ell=1$ ). Using the methods discussed above, we find that

$$
\Phi=\left(\begin{array}{ccc}
1-\frac{1}{d_{1}} & 1 & 1+\frac{1}{d_{1}} \\
1 & 1-\frac{1}{d_{2}} & 1-\frac{2}{d_{2}} \\
1+\frac{1}{d_{1}} & 1-\frac{2}{d_{2}} & 1-\frac{1}{d_{1}}-\frac{4}{d_{2}}
\end{array}\right)
$$

Moreover any two weight vectors span $\mathbb{R}^{2}$. It follows that we need only consider singletons and the full set $\{1,2,3\}$ as candidates for $S$. Applying our algorithm we find the only possibilities are:

$$
S=\{1,2,3\}
$$

and

$$
S=\{3\} \text { provided that } 1-\frac{1}{d_{1}}-\frac{4}{d_{2}}<0,
$$

in agreement with the result of [DW4].

Similarly we may rederive the case when $r=2$ and there are four weight vectors $(\ell=2)$, which was also treated in [DW4].

Remark 2.14. If there are some indices $i$ for which $m_{i}<-2$, we let $\tilde{S}$ denote the subset of such indices corresponding to the minimal leading powers. Equation (2.5) still holds, and in conjunction with (1.4) and the argument of Remark 2.10 this immediately puts some restrictions on $\tilde{S}$.

Now (1.6) implies that $\sum_{j \in \tilde{S}} Q_{j i} \alpha_{0}^{(j)}=0$ for $i=1, \ldots, r$. These $r$ equations are supplemented by the $\ell$ equations on $\alpha_{0}$ coming from the constraints (1.7). In most cases these equations force some or all of the $\alpha_{0}^{(i)}$ to be zero, giving a contradiction. In the examples we consider this is true except in Example (5.2) where $\tilde{S}=\{1,7,8\}$ is possible.

\section{B. Metric Asymptotics}

The convergent Painlevé expansions of Eqs. (1.5)-(1.8) of course give local solutions of the cohomogeneity one Ricci-flat equations. One can easily derive from the leading term behaviour of a Painlevé expansion (as $s$ tends to 0$)$ the behaviour of the corresponding Ricci-flat metric. 
Let $f_{i}(t)^{2}=e^{q_{i}(t)}$ in (1.2). As in [DW4], let $U$ denote the $r \times r$ matrix consisting of the first $r$ rows of $\hat{U}$. Let $\xi$ be the vector $-d U^{-1}$ and $U^{i j}$ denote the components of $U^{-1}$. Recall that the geodesic parameter $t$ is related to the independent variable $s$ of our quadratic system (1.5)-(1.6) by

$$
d s=\exp \left(-\frac{1}{2}(d \cdot q)\right) d t=\prod_{j=1}^{r}\left(\frac{z_{j}}{A_{j}}\right)^{\frac{1}{2} \xi_{j}} d t .
$$

Also,

$$
f_{i}(t)^{2}=\prod_{j=1}^{r}\left(\frac{z_{j}}{A_{j}}\right)^{U^{i j}} .
$$

If we now use the expressions for the leading terms of the Painlevé expansion in the above relations, it follows easily that

(I) if $1-\frac{1}{2} \sum_{i=1}^{r} m_{i} \xi_{i}<0$, then as $s \rightarrow 0^{+}$, we must have $t \rightarrow+\infty$ (after possibly changing the sign of $t$ ), and asymptotically, we have

$$
f_{i}(t)^{2} \sim c_{i} t^{\frac{\sum_{j} U^{i j} m_{j}}{1-\frac{1}{2} \sum_{j} m_{j} \xi_{j}}}
$$

(II) if $1-\frac{1}{2} \sum_{i=1}^{r} m_{i} \xi_{i}>0$, then as $s \rightarrow 0^{+}, t$ decreases to a finite limit $t^{*}$, and asymptotically, we have

$$
f_{i}(t)^{2} \sim c_{i}\left(t-t^{*}\right)^{\frac{\sum_{j} U^{i j} m_{j}}{1-\frac{1}{2} \sum_{j} m_{j} \xi_{j}}}
$$

Note that the constants $c_{i}$ in the above asymptotic formulae are given by

$$
c_{i}=\left(\prod_{j=1}^{r}\left(\frac{\alpha_{0}^{(j)}}{A_{j}}\right)^{U^{i j}}\right)\left(\left|\frac{1}{2}\left(\sum_{j=1}^{r} m_{j} \xi_{j}\right)-1\right| \prod_{j}\left(\frac{\alpha_{0}^{(j)}}{A_{j}}\right)^{\frac{\xi_{j}}{2}}\right)^{\frac{\sum U^{i j} m_{j}}{1-(1 / 2) \sum m_{j} \xi_{j}}} .
$$

Also, $1-\frac{1}{2} \sum_{i} m_{i} \xi_{i}$ cannot be 0 , for otherwise $f_{i}(t)^{2}$ would be asymptotically of the form $\exp \left(-C t \sum_{j} U^{i j} m_{j}\right)$ and since $-d U^{-1} m=2$ in this case, we would have a complete Ricci-flat end with exponential volume growth.

It follows from (I) above that a convergent Painlevé expansion for which $1-\frac{1}{2} \sum_{j} m_{j} \xi_{j}<0$ corresponds to a complete Ricci-flat end, and $f_{i}(t)^{2}$ is asymptotic to a nonzero constant iff $\sum_{j} U^{i j} m_{j}=0$.

The first interesting type of leading term behaviour is when we have $S=\{1, \ldots, r+\ell\}$ and $T=\{1, \ldots, r\}$, that is, all $m_{i}$ equal -2 and all $n_{i}$ 
equal -1 . In this case the leading terms are themselves an exact solution of the equations. Each such solution corresponds to the Ricci-flat cone over a homogeneous Einstein metric on $G / K$.

Painlevé expansions with such leading terms therefore correspond to metrics asymptotic to the cone ( $\mathrm{AC}$ in the terminology of [CGLP1]). We refer to such leading terms as being of cone type. We note further that, in the expression (1.1) for such a Ricci-flat metric, if we let $\tilde{g}_{t}$ denote the unit volume metric in the homothety class of $g_{t}$, then $\tilde{g}_{t}$ is a curve in the space of unit volume $G$-invariant metrics on $G / K$ which converges (in the $C^{\infty}$ topology) to a $G$-invariant Einstein metric with Einstein constant $n-1$. The corresponding complete Ricci-flat ends necessarily have Euclidean volume growth.

A second interesting type of leading term behaviour is the following. In case (I), suppose that the condition $\sum_{j} U^{i j} m_{j}=0$ holds for some $i$ and the corresponding summand $\mathfrak{p}_{i}$ is such that $\mathfrak{k}^{\prime}=\mathfrak{k} \oplus \mathfrak{p}_{i}$ is the Lie algebra of some closed subgroup $K^{\prime}$ of $G$. Suppose further that $\oplus_{j \neq i} \mathfrak{p}_{j}$ and the induced metrics on it are $A d\left(K^{\prime}\right)$-invariant for all $t$ sufficiently large. Finally assume that for all $j \neq i, f_{j}(t)^{2}$ grows like $t^{2}$. Then the principal orbits in the complete Ricci-flat end will be asymptotic to a $K^{\prime} / K$ bundle over $G / K^{\prime}$ with isometric fibres, and the end will have sub-Euclidean volume growth. As is well-known, this happens in the case of the Euclidean Taub-NUT space. Notice that locally (in $t$ ) the metric is asymptotically that of a bundle with isometric fibres over a cone on an Einstein metric on $G / K^{\prime}$.

Recently, such end behaviour has become important in string theory, especially in connection with the construction of complete non-compact manifolds with $G_{2}$ or $\operatorname{Spin}(7)$ holonomy. In [CGLP1] the terminology asymptotically locally conical (or ALC) was introduced to describe such end behaviour. Specifically, the situation when $K^{\prime} / K$ is a circle (i.e., $\mathfrak{p}_{i}$ is a 1-dimensional trivial $K$-representation) of asymptotically constant radius has physical interpretations (cf [BGGG], [CGLP1] and [CGLP2]).

The following proposition puts the ALC condition in a more formal framework and provides a connection to Palais-Smale sequences (cf [St], p. 78) for the Hilbert action on the principal orbit.

Proposition 2.15. Let $K^{\prime}$ be a compact Lie group, $K \subset K^{\prime}$ be a closed subgroup such that $K^{\prime} / K$ is connected, and let $\pi: \mathcal{Q} \rightarrow \mathcal{B}$ be a principal $K^{\prime}$-bundle over a compact base. Denote by $\mathcal{P}$ the bundle associated to $\mathcal{Q}$ with fibre $K^{\prime} / K$. Let $n$ be the dimension of $\mathcal{P}$. Suppose that $\omega$ is a fixed principal connection on $\mathcal{Q}, h_{t}$ is a 1-parameter family of $K^{\prime}$-invariant metrics 
on $K^{\prime} / K$, and $g_{t}{ }^{*}$ is a 1-parameter family of Riemannian metrics on $\mathcal{B}$, where $t$ lies in some open interval $I=\left(t_{0},+\infty\right)$. Let $g_{t}$ be the unique metric on the total space $\mathcal{P}$ induced by $\omega, h_{t}$, and $g_{t}{ }^{*}$.

Assume that $g=d t^{2}+g_{t}$ is a Ricci-flat metric on $\mathcal{M}=I \times \mathcal{P}$. Finally, assume that as $t \rightarrow+\infty$ the following asymptotic conditions are satisfied:

1. $h_{t}=h_{\infty}+t^{-1} \rho+O\left(t^{-2}\right)$,

2. $t^{-2} g_{t}^{*}=g_{\infty}^{*}+t^{-1} \sigma+O\left(t^{-2}\right)$,

where $h_{\infty}, \rho$ are respectively $K^{\prime}$-invariant metrics (symmetric 2 -tensors) on $K^{\prime} / K$ and $g_{\infty}^{*}, \sigma$ are respectively metrics (symmetric 2-tensors) on $\mathcal{B}$.

Then $K^{\prime} / K$ must be a torus and $g_{\infty}^{*}$ is Einstein with scalar curvature $(\operatorname{dim} \mathcal{B})(\operatorname{dim} \mathcal{B}-1)$. Furthermore, if $\tilde{g}_{t}$ denotes the unit volume metric in the homothety class of $g_{t}$, then

$$
\lim _{t \rightarrow+\infty} S\left(\tilde{g}_{t}\right)=0
$$

and

$$
\lim _{t \rightarrow+\infty}\left\|\operatorname{Ric}\left(\tilde{g}_{t}\right)-\frac{S\left(\tilde{g}_{t}\right)}{n} \tilde{g}_{t}\right\|_{L^{2}\left(\tilde{g}_{t}\right)}=0 .
$$

Note that in $(2.17)$ we are taking the $L^{2}$-norm (defined by the metric $\tilde{g}_{t}$ ) of the traceless Ricci tensor of $\tilde{g}_{t}$, which is the negative of the $L^{2}$-gradient of the Hilbert functional on the space of unit volume metrics on $\mathcal{P}$. So for any sequence $\left\{t_{k}\right\}$ going to $+\infty,\left\{\tilde{g}_{t_{k}}\right\}$ is a Palais-Smale sequence of this action.

Proof. We will use the formalism in [DW1] and [EW]. We first choose a background metric $g_{b}$ of volume 1 on $\mathcal{P}$ by choosing a metric on $\mathcal{B}$ and a $K^{\prime}$-invariant metric on $K^{\prime} / K$. We express each $g_{t}$ and its Ricci tensor via $g_{b}$ and $g_{t}$ respectively as symmetric endomorphisms $g_{t}$ and $r_{t}$ of $T \mathcal{P}$. If $V(t)$ denotes the volume of $g_{t}$, then $\tilde{g}_{t}$ is given explicitly by $V(t)^{-(2 / n)} g_{t}$.

It follows from the asymptotic conditions 1 . and 2 . that the shape operator $L$ of $\mathcal{P}$ takes the form

$$
L=\frac{1}{2}\left(\begin{array}{cc}
-t^{-2} h_{\infty}^{-1} \rho+O\left(t^{-3}\right) & 0 \\
0 & \frac{2}{t} I+O\left(t^{-2}\right)
\end{array}\right),
$$

and

$$
V(t)=t^{\operatorname{dim} \mathcal{B}} v_{\infty}^{2 / n}+O\left(t^{\operatorname{dim} \mathcal{B}-\frac{1}{2}}\right)
$$

where

$$
v_{\infty}=\int_{\mathcal{P}} \sqrt{\left(\operatorname{det} h_{\infty}\right)\left(\operatorname{det} g_{\infty}\right)} d v o l_{g_{b}}
$$


Combining the above formulae with the Hamiltonian constraint $H=0$ (cf Remark 1.14 in [DW1]) and using the fact that $\operatorname{dim} \mathcal{B}<n$, it follows readily that $\lim _{t \rightarrow+\infty} S\left(\tilde{g}_{t}\right)=0$. (Indeed, the same computation shows that if $d t^{2}+g_{t}$ is Einstein with constant $\Lambda \neq 0$, this limit is $\pm \infty$ according to the sign of $\Lambda$.)

Similarly, if we use the Einstein equation (1.2) in [DW1], we can verify that the Ricci endomorphisms $r_{t}$ satisfy

$$
r_{t}=\frac{1}{2}\left(\begin{array}{cc}
(2-\operatorname{dim} \mathcal{B}) t^{-3} h_{\infty}^{-1} \rho+O\left(t^{-4}\right) & 0 \\
0 & 2(\operatorname{dim} \mathcal{B}-1) t^{-2} I+O\left(t^{-3}\right)
\end{array}\right) .
$$

Then the conclusion (2.17) follows from a straightforward computation, for which we need to use the fact $\operatorname{dim} \mathcal{B}<n$ once more when we take the limit in $t$.

Next we consider the O'Neill equations for the Riemannian submersion metrics $g_{t}$ (cf Proposition 9.36 in [Bes]). Since the fibres are totally geodesic, all terms involving O'Neill's $T$-tensor vanish. As the fibre metrics are homogeneous, to see that $K^{\prime} / K$ is a torus, it suffices for us to show that $h_{\infty}$ is Ricci-flat, since any Ricci-flat homogeneous metric is automatically flat. In Eq. (9.36a) in [Bes], using (2.20) we see that the left-hand side tends to 0 as $t \rightarrow+\infty$. To compute the terms involving O'Neill's $A$-tensor, we may choose a $g_{\infty}$-orthonormal frame $\left\{E_{i}^{(\infty)}\right\}$ and corresponding orthonormal frames $\left\{E_{i}^{(t)}\right\}$ for the metrics $t^{-2} g_{t}{ }^{*}$ such that $E_{i}^{(t)} \rightarrow E_{i}^{(\infty)}$ as $t \rightarrow+\infty$. Since $\left\{t^{-1} E_{i}^{(t)}\right\}$ are orthonormal with respect to $g_{t}{ }^{*}$, we can use their horizontal lifts in computing the O'Neill $A$-tensor terms. From the asymptotic conditions 1 . and 2 . and the fact that the Lie brackets $\left[t^{-1} E_{i}^{(t)}, t^{-1} E_{j}^{(t)}\right]$ $=t^{-2}\left[E_{i}^{(t)}, E_{j}^{(t)}\right]$ converge to 0 as $t \rightarrow+\infty$, it follows that the O'Neill $A$ tensor term in (9.36a) of [Bes] also tends to zero. Therefore $K^{\prime} / K$ is a torus.

Finally, using (2.20), we see that the left-hand side of (9.36c) tends to $(\operatorname{dim} \mathcal{B}-1) g_{\infty}$. As above, the term involving O'Neill's $A$-tensor on the righthand side tends to zero, while the term involving the Ricci tensor of the base tends to $\operatorname{Ric}\left(g_{\infty}\right)$. Hence $g_{\infty}$ is Einstein with constant $\operatorname{dim} \mathcal{B}-1$ as asserted. The proof of the proposition is now complete.

Let us now return to the cohomogeneity one case where $\mathcal{P}=G / K$.

Remark 2.21. The two consequences (2.16) and (2.17) for the renormalised family $\tilde{g}_{t}$ in the ALC situation should be compared to the condition in the AC case that the family $\tilde{g}_{t}$ converges to a $G$-invariant Einstein metric with 
constant $n-1$. These conditions give an upper bound on the number of parameters arising from leading order considerations in Painlevé expansions of $\mathrm{AC}$ or ALC type.

In the three examples that we will consider in $\S 5$ and $\S 6$, with one exception, all admissible choices of the sets $S$ and $T$ lead to ends which are either incomplete or else of AC or ALC type. The exception occurs in Example 5.2 in the situation where the minimal leading powers are $<-2$, i.e., when Remark (2.14) applies. In that case, we get complete ends asymptotic to a 2-torus bundle (cf Theorem 5.11).

Remark 2.22. Note also that by Theorem 2.1 in [BWZ], the existence on $G / K$ of a sequence of unit volume $G$-invariant metrics satisfying (2.16) and (2.17) is already enough for concluding that $G / K$ is covered by a homogeneous torus bundle. The hypotheses in (2.15) are of course stronger, and hence we were able to deduce more directly that $K^{\prime} / K$ is a torus.

\section{Resonances.}

Next we shall investigate how the leading terms of the previous section can be extended to full series solutions to equations (1.5)-(1.6), in (possibly fractional) powers of $s$. Let us write

$$
\begin{gathered}
z_{i}=\sum_{j=0}^{\infty} \alpha_{j}^{(i)} s^{m_{i}+\frac{j}{N}} \\
v_{i}=\sum_{j=0}^{\infty} \beta_{j}^{(i)} s^{n_{i}+\frac{j}{N}}
\end{gathered}
$$

where $N$ is an integer to be determined after all the resonances have been computed.

The equations (1.5)-(1.6) will now yield recursion relations involving the $\alpha_{j}, \beta_{j}$. Schematically, the recursion may be written as

$$
\mathcal{T}_{j}\left(\alpha_{j}, \beta_{j}\right)=\text { expression in } \alpha_{k}, \beta_{k} \quad(k<j),
$$

where $\mathcal{T}_{j}$ is a linear operator. The rational resonances of the expansion are the values of $j / N$ for which $\mathcal{T}_{j}$ is non-invertible; that is, they give the stages in the recursion at which free parameters may enter. In this section we aim to calculate the resonances of the various Painlevé expansions to our equations. We shall continue to assume that $m_{i} \geq-2$ for all $i$. 
We substitute the full expansions (3.1)-(3.2) into the equations (1.5)(1.6). Equating coefficients of $s^{m_{i}+\frac{j}{N}-1}$ in (1.5), and $s^{n_{p}+\frac{j}{N}-1}$ in (1.6), we obtain the recursion relations

$$
\left(m_{i}+\frac{j}{N}\right) \alpha_{j}^{(i)}=\sum_{p=1}^{r} \sum_{k=0}^{j-N\left(n_{p}+1\right)} 2 \alpha_{k}^{(i)} Q_{i p} \beta_{j-k-N\left(n_{p}+1\right)}^{(p)}
$$

and

$$
\left(n_{p}+\frac{j}{N}\right) \beta_{j}^{(p)}=\epsilon_{p} \sum_{i=1}^{r+\ell} Q_{i p} \alpha_{j-N\left(m_{i}-n_{p}+1\right)}^{(i)} .
$$

Using (2.5) we may rewrite (3.3) as

$$
\begin{aligned}
\frac{j}{N} \alpha_{j}^{(i)}-2 \alpha_{0}^{(i)}\left(\sum_{p \in T} Q_{i p} \beta_{j}^{(p)}\right)= & \sum_{p \in T} \sum_{k=1}^{j-1} 2 Q_{i p} \alpha_{k}^{(i)} \beta_{j-k}^{(p)} \\
& +\sum_{p \notin T}^{j-N\left(n_{p}+1\right)} \sum_{k=0}^{j(3.5)} 2 Q_{i p} \alpha_{k}^{(i)} \beta_{j-k-N\left(n_{p}+1\right)}^{(p)}
\end{aligned}
$$

If $p \in T$, we can write (3.4) as

$$
\left(\frac{j}{N}-1\right) \beta_{j}^{(p)}-\epsilon_{p} \sum_{i=1}^{r+\ell} P_{i p} \alpha_{j}^{(i)}=\epsilon_{p} \sum_{i \notin S} Q_{i p} \alpha_{j-N\left(m_{i}+2\right)}^{(i)} .
$$

We refer to the expressions on the right-hand side of (3.5) and (3.6) respectively as $X_{j}^{(i)}$ and $Y_{j}^{(p)}$. Note that these quantities involve only terms with subscripts $<j$ (because $n_{p} \geq-1$ with equality iff $p \in T$, and because by assumption $m_{i} \geq-2$ with equality iff $\left.i \in S\right)$. Moreover, the terms $\beta_{j}^{(p)}$ with $p \notin T$ do not appear on the right-hand side.

We may therefore first consider equations (3.5)-(3.6), which form a subsystem of the full recursion. Eliminating $\alpha_{j}$ by (3.5) we obtain, for $p \in T$,

$$
\frac{j}{N}\left(\frac{j}{N}-1\right) \beta_{j}^{(p)}-2 \epsilon_{p} \sum_{i} \sum_{q \in T} P_{i p} \alpha_{0}^{(i)} Q_{i q} \beta_{j}^{(q)}=Z_{j}^{(p)}
$$

where $Z_{j}^{(p)}=\frac{j}{N} Y_{j}^{(p)}+\epsilon_{p} \sum_{i} P_{i p} X_{j}^{(i)}$ involves only terms with subscripts $<j$.

Let $M:=2 D P^{t} \operatorname{diag}\left(\alpha_{0}^{(1)}, \ldots, \alpha_{0}^{(r+\ell)}\right) Q$, where $P^{t}$ denotes the transpose of $P$, and let us introduce the $|T| \times|T|$ matrix $M_{T}$ obtained from $M$ by 
deleting the $p$ th row and $p$ th column for all $p$ not in $T$. Equation (3.7) is then just

$$
\left(\frac{j}{N}\left(\frac{j}{N}-1\right)-M_{T}\right) \beta_{j}=Z_{j}
$$

In other words, $\beta_{j}^{(p)}(p \in T)$ and $\alpha_{j}$ are uniquely determined by the earlier terms $\alpha_{k}, \beta_{k}(k<j)$ unless $R=j / N$ satisfies the condition that

$$
R(R-1) \text { is an eigenvalue of } M_{T} .
$$

Such $R$ are the rational resonances of the subsystem (3.5)-(3.6), while the resonances refer to real (or even complex) solutions of (3.9).

Remark 3.10. Note that these resonances only depend on $\alpha_{0}$ via the components $\alpha_{0}^{(i)}$ for $i \in S$, i.e., the vector $\hat{\alpha}_{0}$.

Remark 3.11. It is easy to check from (2.5) and (2.6) that $\hat{\beta}_{0}$ is an eigenvector of $M$ with eigenvalue 2 . It follows that $\left(\beta_{0}^{(p)}\right)_{p \in T}$ is an eigenvector of $M_{T}$ with eigenvalue 2 , and hence that

$$
-1,2 \text { are resonances. }
$$

Note that

$$
\left(\begin{array}{c}
\left(\frac{1}{2} m_{i} \alpha_{0}^{(i)}\right)_{i=1}^{r+\ell} \\
\left(\beta_{0}^{(p)}\right)_{p \in T}
\end{array}\right)
$$

lies in the kernel of the recursion (3.5)-(3.6) for $R=2$ (that is, $j=2 N$ ). We shall see in the next section that the resonance $R=2$ is connected with the Hamiltonian constraint (1.8).

The appearance of -1 as a resonance is typical for autonomous systems of differential equations, and is associated to the degree of freedom implicit in translating the independent variable and hence changing the position of the singularity.

It often happens that $-1,2$ are the only rational resonances, and that the free parameter at $R=2$ is fixed by the Hamiltonian constraint. In this case translation of $s$ is the only free parameter in the Painlevé expansion and we refer to such expansions as trivial.

Existence of nontrivial Painlevé expansions requires the existence of a rational root $R$ of (3.9) other than $-1,2$. This can impose constraints on the principal orbit $G / K$, and in particular can lead to Diophantine conditions involving, for example, the dimension of $G / K$. An example is discussed in $\S 6$. 
Finally, let us consider the situation for $\beta_{j}^{(p)}(p \notin T)$. In most of our examples, the following conditions hold.

(i) $m_{i} \geq n_{p}-1$ for all $p \notin T$ and $i \notin S$ with $Q_{i p} \neq 0$,

(ii) $Q_{i p}=0$ for all $p \notin T$ and $i \in S$.

Condition (ii) means that, for $p \notin T$, equation (3.4) reduces to

$$
\left(n_{p}+\frac{j}{N}\right) \beta_{j}^{(p)}=\epsilon_{p} \sum_{i \notin S} Q_{i p} \alpha_{j-N\left(m_{i}-n_{p}+1\right)}^{(i)} .
$$

We observe that condition (i) now shows that $\beta_{j}^{(p)}$, with $p \notin T$, are uniquely determined by $\alpha_{k}(k \leq j)$ unless $\frac{j}{N}=-n_{p}$, so the only additional resonance arising from (3.12) is $R=-n_{p}$.

Remark 3.13. The conditions (i),(ii) do not have to hold. This is the situation for instance in the case $S=\{4,5\}$ in Example (5.2). In this case we can perform a reindexing $j \mapsto j-N\left(n_{p}+1\right)$ for the terms $\beta_{j}^{(p)}$ where $p \notin T$, and bring the system back into the form considered above.

Remark 3.14. Equations (3.5)-(3.6), (3.12) are valid for $j>0$. We may also have additional zero resonances, associated to free parameters in $\alpha_{0}, \beta_{0}$.

\section{Compatibility conditions.}

As remarked earlier, the resonances correspond to the steps in the recursion at which the recursion operator is non-invertible. This means that at each resonance there are compatibility conditions which must be satisfied if the recursion is to proceed. These conditions express the fact that the righthand side of the recursion should be in the image of the recursion operator. These conditions may put constraints on the free parameters introduced at earlier resonances.

In all our examples compatibility holds at the top resonance $R=2$. In many of these cases we can see this from the following proposition.

Proposition 4.1. Suppose that all compatibility conditions hold for $R=$ $\frac{j}{N}<2$, and we have

(i) $m_{i} \geq n_{p}-1$ for all $p \notin T, i \notin S$ with $Q_{i p} \neq 0$,

(ii) $Q_{i p}=0$ for all $p \notin T$ and $i \in S$,

(iii) the 2-eigenspace of $M_{T}$ is one-dimensional. Suppose in addition that either

(iv) $m_{i}+n_{p}+1>0$ for all $p \notin T$ and $i \notin S$ with $Q_{i p} \neq 0$, or 
(v) $m_{i}-n_{p}+1=0$ for all $p \notin T$ and $i \notin S$ with $Q_{i p} \neq 0$.

Then we can solve the recursion at $R=2$.

Proof. Assumptions (i) and (ii) show, by the discussion of $\S 3$, that solvability of the recursion at $R=2$ (that is, $j=2 N$ ) is equivalent to solvability of (3.8) at $j=2 N$.

It is straightforward to check, using (2.5) and (2.6), that $D \hat{\beta}_{0}$ is an eigenvector of $M^{t}$ with eigenvalue 2 , and hence the truncated column vector $\left(\left(D \beta_{0}\right)_{p \in T}\right)$ is an eigenvector of $M_{T}^{t}$, also with eigenvalue 2. Assumption (iii) means that this vector spans the eigenspace, so we have a single compatibility condition for (3.8). After some further calculation using (2.5) this condition becomes

$$
-\sum_{i \in S} X_{2 N}^{(i)}+2 \sum_{p \in T} \epsilon_{p} \beta_{0}^{(p)} Y_{2 N}^{(p)}=0
$$

where $X_{j}^{(i)}, Y_{j}^{(p)}$ were defined in $\S 3$.

Assumption (ii) now shows that the first sum in (4.2) simplifies to

$$
-2 \sum_{p \in T} \sum_{k=1}^{2 N-1}\left(P^{t} \alpha_{k}\right)^{(p)} \beta_{2 N-k}^{(p)}
$$

which, using (3.6), becomes

$$
-2 \sum_{p \in T} \epsilon_{p} \sum_{k=1}^{2 N-1}\left(\left(\frac{k}{N}-1\right) \beta_{k}^{(p)} \beta_{2 N-k}^{(p)}-Y_{k}^{(p)} \beta_{2 N-k}^{(p)}\right) .
$$

We can use the index change $q=2 N-k$ to observe that the sum $\sum_{k=1}^{2 N-1}\left(\frac{k}{N}-\right.$ 1) $\beta_{k}^{(p)} \beta_{2 N-k}^{(p)}$ is zero. Hence the compatibility condition becomes

$$
\sum_{p \in T} \epsilon_{p} \sum_{k=1}^{2 N} Y_{k}^{(p)} \beta_{2 N-k}^{(p)}=0 .
$$

As $Y_{k}^{(p)}=\epsilon_{p} \sum_{j \notin S} Q_{p j}^{t} \alpha_{k-N\left(m_{j}+2\right)}^{(j)}$ (for $\left.p \in T\right)$, (4.3) becomes

$$
\sum_{j \notin S} \sum_{p \in T} \sum_{k=1}^{2 N} \alpha_{k-N\left(m_{j}+2\right)}^{(j)} Q_{j p} \beta_{2 N-k}^{(p)}=0 .
$$

Now the sum over $\{j \notin S\}$ in (4.4) is only a sum over $\left\{j \notin S: m_{j}<0\right\}$ because if $m_{j} \geq 0$ and $j \notin S$ then we only get a contribution from terms with $k=2 N$ and $m_{j}=0$. This contribution is zero by (2.2). 
For each $j \notin S$ with $m_{j}<0$, we have $0<-N m_{j}<2 N$. If we let $i=k-N\left(m_{j}+2\right)$, then

$$
\sum_{p \in T} \sum_{k=1}^{2 N} \alpha_{k-N\left(m_{j}+2\right)}^{(j)} Q_{j p} \beta_{2 N-k}^{(p)}=\sum_{p \in T} \sum_{i=0}^{-N m_{j}} \alpha_{i}^{(j)} Q_{j p} \beta_{-N m_{j}-i}^{(p)} .
$$

Using the recursion relation (3.5), in which we replace $i$ by $j$ and $j$ by $-N m_{j}$, the above becomes

$$
-\sum_{p \notin T} \sum_{i=0}^{-N\left(m_{j}+n_{p}+1\right)} \alpha_{i}^{(j)} Q_{j p} \beta_{-N\left(m_{j}+n_{p}+1\right)-i}^{(p)} .
$$

We may substitute this last expression back into (4.4), noting that for those $j \notin S$ with $m_{j} \geq 0$, the factor $m_{j}+n_{p}+1$ in the upper summation index is positive since $p \notin T$. Hence our compatibility condition (4.4) is just

$$
\sum_{j \notin S} \sum_{p \notin T} \sum_{i=0}^{-N\left(m_{j}+n_{p}+1\right)} \alpha_{i}^{(j)} Q_{j p} \beta_{-N\left(m_{j}+n_{p}+1\right)-i}^{(p)}=0 .
$$

The case of our proposition when condition (iv) holds follows immediately from this formula.

To study the situation when (v) holds, note first that the left-hand side of (4.5) now becomes

$$
\sum_{j \notin S} \sum_{p \notin T} \sum_{i=0}^{-2 N n_{p}} \alpha_{i}^{(j)} Q_{j p} \beta_{-2 N n_{p}-i}^{(p)} .
$$

We next perform the summation over $j$ first with the help of the recursion relation (3.12), and get

$$
\sum_{p \notin T} \sum_{i=0}^{-2 N n_{p}} \epsilon_{p}\left(n_{p}+\frac{i}{N}\right) \beta_{i}^{(p)} \beta_{-2 N n_{p}-i}^{(p)} .
$$

This expression is zero by the same argument as before where we replace the index $i$ by $-2 N n_{p}-i$ and the sum changes sign.

Remark 4.6. Note that the condition (iv) holds, for example, if $m_{i} \geq 0$ for all $i \notin S$. This condition occurs frequently in the examples in $\S 5$ and $\S 6$. Condition (iv) also holds if $n_{p}>0$ for all $p \notin T$ since we are assuming (i). Condition (v) holds, for example, if there exist constants $\nu_{1}$ and $\nu_{2}$ such that $\nu_{1}-\nu_{2}+1=0$ and $m_{i}=\nu_{1}$ for all $i \notin S, n_{p}=\nu_{2}$ for all $p \notin T$. This condition will hold in the ALC cases of Examples 5.2 and 6.1. 
We shall also discuss the constraints. As $z_{r+j} \prod_{i=1}^{r} z_{i}^{-\nu_{j i}}(j=1, \ldots, \ell)$ are constants of the flow, and we have $m_{j+r}=\sum_{k=1}^{r} \nu_{j k} m_{k}$ resulting from (1.4) and (2.2), the constraints (1.7) are equivalent to requiring that $\alpha_{0}$ satisfies

$$
\frac{\alpha_{0}^{(r+j)}}{A_{r+j}}=\prod_{i=1}^{r}\left(\frac{\alpha_{0}^{(i)}}{A_{i}}\right)^{\nu_{j i}}: \quad(1 \leq j \leq \ell) .
$$

The Hamiltonian constraint is related to the resonance at $R=2$, as we now explain. After substituting the Painlevé expansion into the Hamiltonian (1.8), one sees that the constant term is given by the expression

$$
2 \sum_{p \in T} \epsilon_{p} \beta_{0}^{(p)} \beta_{2 N}^{(p)}-\sum_{i \in S} \alpha_{2 N}^{(i)}+\text { terms with subscripts }<2 N .
$$

As remarked in (3.11) the vector

$$
\left(\begin{array}{c}
\alpha \\
\beta
\end{array}\right)=\left(\begin{array}{c}
\left(\frac{1}{2} m_{i} \alpha_{0}^{(i)}\right)_{i=1}^{r+\ell} \\
\left(\beta_{0}\right)_{p \in T}
\end{array}\right)
$$

is in the kernel of the recursion operator at $R=2$, that is, at $j=2 N$. We therefore deduce

Proposition 4.8. If all compatibility conditions hold at $R=2$, and if

$$
2 \sum_{p \in T} \epsilon_{p}\left(\beta_{0}^{(p)}\right)^{2}+\sum_{i \in S} \alpha_{0}^{(i)} \neq 0
$$

then the Hamiltonian constraint may always be satisfied by choosing $\alpha_{2 N}, \beta_{2 N}$ appropriately. In this situation the Hamiltonian constraint fixes one of the degrees of freedom coming from the resonance $R=2$.

\section{Some 7-dimensional examples and $G_{2}$ metrics.}

Example 5.1. Principal orbit $S U(3) / T^{2}$.

The isotropy representation is the sum of the three 2-dimensional real root spaces of $S U(3)$, which are permuted by the action of the Weyl group, the symmetric group on three letters. In the scalar curvature formula, the weight vectors $w^{(1)}, \ldots, w^{(6)}$ are respectively

$$
(-1,0,0),(0,-1,0),(0,0,-1),(1,-1,-1),(-1,1,-1),(-1,-1,1),
$$


and the constants $A_{i}$ are given by

$$
\left(A_{1}, \ldots, A_{6}\right)=(6,6,6,-1,-1,-1)
$$

where the background metric is induced by $-\operatorname{tr}(X Y)$.

So $r=\ell=3$ and our equations are a 9-dimensional system with three constraints (together with the Hamiltonian constraint). The matrix $\Phi$ and a choice for $Q$ are as follows.

$$
\Phi=\left(\begin{array}{rrrrrr}
\frac{1}{2} & 1 & 1 & \frac{3}{2} & \frac{1}{2} & \frac{1}{2} \\
1 & \frac{1}{2} & 1 & \frac{1}{2} & \frac{3}{2} & \frac{1}{2} \\
1 & 1 & \frac{1}{2} & \frac{1}{2} & \frac{1}{2} & \frac{3}{2} \\
\frac{3}{2} & \frac{1}{2} & \frac{1}{2} & -\frac{1}{2} & \frac{3}{2} & \frac{3}{2} \\
\frac{1}{2} & \frac{3}{2} & \frac{1}{2} & \frac{3}{2} & -\frac{1}{2} & \frac{3}{2} \\
\frac{1}{2} & \frac{1}{2} & \frac{3}{2} & \frac{3}{2} & \frac{3}{2} & -\frac{1}{2}
\end{array}\right), \quad Q=\left(\begin{array}{rrr}
\frac{1}{\sqrt{2}} & 0 & 0 \\
\sqrt{2} & \frac{\sqrt{3}}{\sqrt{2}} & 0 \\
\sqrt{2} & \frac{\sqrt{2}}{\sqrt{3}} & \frac{\sqrt{5}}{\sqrt{6}} \\
\frac{3}{\sqrt{2}} & \frac{5}{\sqrt{6}} & \frac{\sqrt{5}}{\sqrt{6}} \\
\frac{1}{\sqrt{2}} & -\frac{1}{\sqrt{6}} & \frac{\sqrt{5}}{\sqrt{6}} \\
\frac{1}{\sqrt{2}} & \frac{1}{\sqrt{6}} & -\frac{\sqrt{5}}{\sqrt{6}}
\end{array}\right) .
$$

We find that the possible $S$ are

$$
\{4\},\{5\},\{6\} \text { and }\{1, \ldots, 6\} \text {. }
$$

There are no leading terms with exponent $<-2$.

(I) The three singleton possibilities for $S$ are equivalent because of the action of the Weyl group. If we take $S=\{4\}$ we obtain

$$
m=(6,2,2,-2,6,6), \quad T=\{1,2,3\}, \quad \alpha_{0}^{(4)}=-2 .
$$

We find that the resonances are $-1,0$ (twice), 1 (twice), 2 . The compatibility conditions are all satisfied, giving a Painlevé family depending on the full number of parameters. The quantity $1-\frac{1}{2} \sum_{j} m_{j} \xi_{j}=3>0$ and so as $s$ tends to 0 , the volume of the principal orbits tends to 0 . On the other hand, we can also check that $f_{1}(t)^{2} \sim\left(t-t^{*}\right)^{-2 / 3}$ while $f_{i}(t)^{2} \sim\left(t-t^{*}\right)^{2 / 3}$ for $i=2,3$.

(II) The remaining case is

$$
S=\{1, \ldots, 6\}, \quad m=(-2, \ldots,-2), \quad T=\{1,2,3\} .
$$

We have four possibilities for the leading coefficients, corresponding, as in Remark 2.21, to the three homogeneous Kähler-Einstein metrics and the normal homogeneous Einstein metric on $S U(3) / T^{2}$. As the Kähler-Einstein metrics are equivalent under the Weyl group action, it is sufficient to just 
consider one, which we call case (a). We call the normal homogeneous case (b).

The corresponding expressions for $\alpha_{0}$ are:

(a) $\alpha_{0}=\frac{1}{20}(12,12,6,-1,-1,-4)$,

(b) $\alpha_{0}=\frac{1}{25}(12,12,12,-2,-2,-2)$.

and the resonances are as follows:

(a) $-1,2$ and the roots of $R(R-1)=\frac{1}{5}$ and $R(R-1)=-\frac{2}{5}$ (all of which are irrational).

(b) $-1, \frac{1}{5}$ (twice), $\frac{4}{5}$ (twice), 2 .

Therefore, case (a) only gives a trivial Painlevé family.

In case (b) we find that the compatibility condition at $R=4 / 5$ forces the free parameters at $R=1 / 5$ to be zero. The upshot is that we have a 3-parameter Painlevé family for the Ricci-flat equations. One parameter comes from $R=-1$ (the position of the singularity), and the other two come from $R=\frac{4}{5}$, where the recursion operator has a 2-dimensional kernel.

The cohomogeneity 1 metrics with $G_{2}$-holonomy are given by the solutions of (1.5)-(1.8) which lie in the subvariety defined by the equations

$$
\begin{aligned}
\frac{-1}{\sqrt{3}} v_{2}-\frac{1}{\sqrt{15}} v_{3} & = \pm 2 \sqrt{-z_{4}} \\
v_{1}+\frac{2}{\sqrt{3}} v_{2}-\frac{1}{\sqrt{15}} v_{3} & = \pm 2 \sqrt{-z_{5}} \\
v_{1}+\frac{2}{\sqrt{3}} v_{2}+\frac{4}{\sqrt{15}} v_{3} & = \pm 2 \sqrt{-z_{6}},
\end{aligned}
$$

where the \pm sign depends on the choice of orientation of the $G_{2}$-structure.

Using these equations, one can check that the metrics corresponding to the above 3-parameter family of Painlevé expansions all have holonomy equal to $G_{2}$. A 2-parameter subfamily of these metrics was found by Bryant and Salamon [BS] and Gibbons-Page-Pope [GPP]. The full 3-parameter family was found independently first by Cleyton and Swann [CS], and shortly after by the authors [DW5] and by Cvetič-Gibbons-Lü-Pope [CGLP2].

These $G_{2}$-metrics have a particularly nice description if we write them in the form

$$
F_{1} F_{2} F_{3} d \rho^{2}+\left.\frac{F_{2} F_{3}}{F_{1}} B\right|_{\mathfrak{p}_{1}}+\left.\frac{F_{3} F_{1}}{F_{2}} B\right|_{\mathfrak{p}_{2}}+\left.\frac{F_{1} F_{2}}{F_{3}} B\right|_{\mathfrak{p}_{3}}
$$

where $\rho$ is a new radial coordinate satisfying $\sqrt{F_{1} F_{2} F_{3}} d \rho=d t$ and $t$ is arclength. The equations for $G_{2}$-holonomy then become the $S U(2) \mathrm{Nahm}$ 
equations

$$
\frac{d F_{1}}{d \rho}=F_{2} F_{3}
$$

and cyclically, a well-known integrable system which is solvable in terms of Jacobi functions. The metrics of [BS], [GPP] arise if two of the $F_{i}$ have equal magnitude. The special case when $F_{1}=F_{2}=F_{3}$ is the cone over the normal metric on $S U(3) / T^{2}$ discovered in [B].

These equations are identical to those arising from four-dimensional hyperKähler metrics of cohomogeneity one with respect to a triholomorphic $S U(2)$ action (cf [BGPP]).

A summary of the results for this example was given in [DW5].

Example 5.2. Principal orbit $S^{3} \times S^{3} \approx((S U(2) \times S U(2) \times \Delta U(1)) \ltimes$ $\left.\mathbb{Z}_{2}\right) /\left(\Delta U(1) \times \mathbb{Z}_{2}\right)$.

This example was considered in [BGGG]. The group $\operatorname{Spin}(4) \approx$ $S U(2) \times S U(2)$ acts on each $S^{3}$ factor by left and right translations. The $S U(2) \times S U(2)$ in $G$ is the direct product of the groups of left translations on each $S^{3}$ factor. The $U(1)$ in $G$ is the diagonal circle subgroup of the group $S U(2) \times S U(2)$ of right translations on $S^{3} \times S^{3}$. The $\mathbb{Z}_{2}$ in $G$ is the outer automorphism that interchanges the two $S^{3}$ factors.

We have the inclusions

$$
\mathfrak{k} \hookrightarrow \Delta \mathfrak{u}(1) \oplus \Delta \mathfrak{u}(1) \hookrightarrow(\mathfrak{u}(1) \oplus \mathfrak{u}(1)) \oplus \Delta \mathfrak{u}(1) \hookrightarrow \mathfrak{s u}(2) \oplus \mathfrak{s u}(2) \oplus \Delta \mathfrak{u}(1)
$$

whose successive quotients give the decomposition of the isotropy representation $\mathfrak{p}$ into four irreducible summands of dimensions 1,1,2 and 2. Note that $\mathfrak{p}_{1}$, given by the first quotient, is a trivial representation, while the third quotient gives the direct sum of $\mathfrak{p}_{3}$ and $\mathfrak{p}_{4}$, the 2-dimensional representations. Also, the action of $\mathbb{Z}_{2}$ is nontrivial only on $\mathfrak{p}_{2}$ and $\mathfrak{p}_{4}$, and the action of $\Delta U(1)$ is non-trivial only on $\mathfrak{p}_{3}$ and $\mathfrak{p}_{4}$. We label the weight vectors occurring in the scalar curvature function as

$$
\begin{aligned}
& w^{(1)}=(0,-1,0,0), w^{(2)}=(0,0,-1,0), w^{(3)}=(0,0,0,-1), w^{(4)}=(1,0,-2,0), \\
& w^{(5)}=(1,0,0,-2), w^{(6)}=(0,1,-1,-1), w^{(7)}=(0,-1,1,-1), w^{(8)}=(0,-1,-1,1),
\end{aligned}
$$

and the corresponding coefficients are $\left(A_{1}, \ldots, A_{8}\right)=\left(2,4,4,-\frac{1}{4},-\frac{1}{4},-1,-1,-1\right)$, where the background metric $B$ is induced by the direct sum of the trace forms $-\operatorname{tr}(X Y)$ on the $S U(2)$ factors. 
We see that $r=\ell=4$, and $\Phi$ and a choice of $Q$ are given by

$$
\Phi=\left(\begin{array}{rrrrrrrr}
0 & 1 & 1 & 1 & 1 & 2 & 0 & 0 \\
1 & \frac{1}{2} & 1 & 0 & 1 & \frac{1}{2} & \frac{3}{2} & \frac{1}{2} \\
1 & 1 & \frac{1}{2} & 1 & 0 & \frac{1}{2} & \frac{1}{2} & \frac{3}{2} \\
1 & 0 & 1 & -2 & 0 & 0 & 2 & 0 \\
1 & 1 & 0 & 0 & -2 & 0 & 0 & 2 \\
2 & \frac{1}{2} & \frac{1}{2} & 0 & 0 & -1 & 2 & 2 \\
0 & \frac{3}{2} & \frac{1}{2} & 2 & 0 & 2 & -1 & 1 \\
0 & \frac{1}{2} & \frac{3}{2} & 0 & 2 & 2 & 1 & -1
\end{array}\right), Q=\left(\begin{array}{rrrr}
\sqrt{2} & \sqrt{2} & 0 & 0 \\
\frac{1}{\sqrt{2}} & 0 & 0 & 0 \\
\sqrt{2} & \frac{1}{\sqrt{2}} & 1 & 0 \\
0 & -\frac{1}{\sqrt{2}} & -\frac{1}{2} & \frac{\sqrt{5}}{2} \\
\sqrt{2} & \frac{1}{\sqrt{2}} & \frac{3}{2} & \frac{\sqrt{5}}{2} \\
\frac{1}{\sqrt{2}} & -\frac{1}{\sqrt{2}} & 1 & 0 \\
\frac{3}{\sqrt{2}} & \frac{3}{\sqrt{2}} & 1 & 0 \\
\frac{1}{\sqrt{2}} & \frac{1}{\sqrt{2}} & -1 & 0
\end{array}\right) .
$$

With some amount of computation it is actually possible to determine explicitly all the $G$-invariant Einstein metrics on $G / K$. These will give the leading terms of cone type in our Painlevé expansions. Up to isometry there are only two $G$-invariant Einstein metrics: the product metric on $S^{3} \times S^{3}$, and the metric induced by the normal homogeneous metric via the diffeomorphism $S^{3} \times S^{3} \approx(S U(2) \times S U(2) \times S U(2)) / \Delta(S U(2))$. (The latter Einstein metric was observed, e.g., in [WZ] (cf Proposition 5.5).)

We will first consider Painlevé expansions in which the leading exponent in each $z_{i}$ is $\geq-2$. The one case in which a leading exponent $<-2$ can occur will be discussed at the end of this section.

Applying the algorithm of $\S 2$ we compile the possibilities for $S$ and $T$, and the associated vectors $m$ and $\alpha_{0}$ in the table below.

\begin{tabular}{|c|c|c|c|}
\hline $\mathrm{S}$ & $\mathrm{T}$ & $\mathrm{m}$ & $\alpha_{0}^{(i)}(i \in S)$ \\
\hline$\{5\}$ & $\{1, \ldots, 4\}$ & $(1,1,0,0,-2,0,0,2)$ & $-\frac{1}{2}$ \\
$\{4,6\}$ & $\{1, \ldots, 4\}$ & $(5,1,2,-2,0,-2,6,4)$ & $-\frac{1}{2},-1$ \\
$\{4,8\}$ & $\{1, \ldots, 4\}$ & $(1,1,4,-2,4,4,4,-2)$ & $-\frac{1}{2},-1$ \\
$\{5,6\}$ & $\{1, \ldots, 4\}$ & $(5,2,1,0,-2,-2,4,6)$ & $-\frac{1}{2},-1$ \\
$\{5,7\}$ & $\{1, \ldots, 4\}$ & $(1,4,1,4,-2,4,-2,4)$ & $-\frac{1}{2},-1$ \\
$\{4,5,6\}$ & $\{1, \ldots, 4\}$ & $(6,2,2,-2,-2,-2,6,6)$ & $-\frac{1}{2},-\frac{1}{2},-1$ \\
$\{1, \ldots, 8\}$ & $\{1, \ldots, 4\}$ & $(-2, \ldots,-2)$ & see below \\
$\{4,5\}$ & $\{1,3,4\}$ & $(2,1,1,-2,-2,0,2,2)$ & $-\frac{1}{2},-\frac{1}{2}$ \\
$\{4\}$ & $\{2,3,4\}$ & $(1,0,1,-2,0,0,2,0)$ & $-\frac{1}{2}$ \\
$\{6\}$ & $\{1,2,3\}$ & $(4,1,1,0,0,-2,4,4)$ & -1 \\
$\{7\}$ & $\{1,2,3\}$ & $(0,3,1,4,0,4,-2,2)$ & -1 \\
$\{8\}$ & $\{1,2,3\}$ & $(0,1,3,0,4,4,2,-2)$ & -1 \\
$\{1,2,3,6,7,8\}$ & $\{1,2,3\}$ & $\left(-2,-2,-2,-\frac{3}{2},-\frac{3}{2},-2,-2,-2\right)$ & see below \\
\hline
\end{tabular}

In the above we have not yet imposed the constraints (4.7). 
When $S=\{1, \ldots, 8\}$ we have two possible sets of leading coefficients satisfying the constraints, corresponding respectively to the product and normal homogeneous Einstein metrics on the principal orbit. These are

(a) $\alpha_{0}=\frac{1}{5}\left(2,4,4,-\frac{1}{2},-\frac{1}{2},-1,-1,-1\right)$,

(b) $\alpha_{0}=\frac{9}{25}\left(\frac{2}{3}, 4, \frac{4}{3},-\frac{1}{2},-\frac{1}{18},-1,-\frac{1}{9},-1\right)$.

If $S=\{1,2,3,6,7,8\}$ the only real, nonzero leading coefficients satisfying the constraints are given by

$$
\alpha_{0}=\left(\frac{9}{32}, \frac{3}{4}, \frac{3}{4}, \omega_{0}, \omega_{0},-\frac{1}{4},-\frac{9}{64},-\frac{9}{64}\right)
$$

where $\omega_{0}<0$ is a free parameter.

Regarding asymptotics, the quantity $1-\frac{1}{2} \sum_{j} m_{j} \xi_{j}$ is negative when $S=\{1,2,3,6,7,8\}$ or when $S=\{1, \ldots, 8\}$, and is positive in the remaining cases.

If $S=\{1, \ldots, 8\}$ we are in the AC case. When $S=\{1,2,3,6,7,8\}$, the quantity $\sum_{j} U^{i j} m_{i}$ is zero for $i=1$ only. Recall that $\mathfrak{p}_{1}$ is the trivial summand in the isotropy representation of $G / K$. We can also check that $f_{i}(t)^{2} \sim t^{2}$ for $i=2,3,4$. So in this case we will get complete Ricci-flat ends with ALC asymptotics (cf $\S 2$ ).

For the remaining cases, as $s$ tends to 0 , the volume of the principal orbits tends to 0 , even though at least one $f_{i}(t)^{2}$ blows up. Furthermore, when $S=\{4\},\{5\},\{6\},\{7\},\{8\}$ or $\{4,5\}$, there is also at least one $f_{i}(t)^{2}$ which converges to a positive constant.

We can now compute $M_{T}$ and its eigenvalues, and find the resonances. Recall that only the components $\alpha_{0}^{(i)}$ for $i \in S$ affect the resonances. The results are summarised below. 


\begin{tabular}{|c|c|c|}
\hline $\mathrm{S}$ & Resonances (multiplicities) & $\begin{array}{c}\text { number of } \\
\text { parameters } \\
\text { in expansion }\end{array}$ \\
\hline$\{5\}$ & $-1,0$ (3 times), 1 (3 times), 2 & 7 \\
$\{4,6\}$ & -1 (twice), 0 (twice), 1 (twice), 2 (twice) & 6 \\
$\{4,8\}$ & -1 (twice), 0 (twice), 1 (twice), 2 (twice) & 6 \\
$\{5,6\}$ & -1 (twice), 0 (twice), 1 (twice), 2 (twice) & 6 \\
$\{5,7\}$ & -1 (twice), 0 (twice), 1 (twice), 2 (twice) & 6 \\
$\{4,5,6\}$ & -1 (three times), $0,1,2$ (three times) & 5 \\
$\{1, \ldots, 8\}($ a) & $-1,2$ and roots of $R(R-1)=\frac{2}{5}$ (twice), $R(R-1)=\frac{4}{5}$ & 1 \\
$\{1, \ldots, 8\}(\mathrm{b})$ & $-1, \frac{2}{5}, \frac{3}{5}, 2$ and roots of $R(R-1)=\frac{1}{25}(31 \pm \sqrt{145})$ & 3 \\
$\{4,5\}$ & -1 (twice), 0 (twice), 1 (twice), $2($ twice) & 6 \\
$\{4\}$ & $-1,0\left(3\right.$ times), 1 (twice), 2 and $-n_{1}$ & 7 \\
$\{6\}$ & $-1,0(3$ times), 1 (twice), 2 and 0 or -1 & 7 \\
$\{7\}$ & $-1,0(3$ times), 1 (twice), 2 and 0 or -1 & 7 \\
$\{8\}$ & $-1,0$ (3 times), 1 (twice), 2 and 0 or -1 & 7 \\
$\{1,2,3,6,7,8\}$ & $-1,-\frac{1}{2}, 0, \frac{1}{4}, \frac{3}{4}, \frac{3}{2}, 2$ and $\frac{1}{2}$ & 4 \\
\hline
\end{tabular}

Remark 5.3. (i) In the cases where $|T|<4$, (except for $\{4,5\}$ for which see Remark 3.13) the resonances listed after "and" arise from the possible values of $-n_{p}(p \notin T)$. When $S=\{4\}, n_{1}=0$ or $n_{1} \geq 1$.

(ii) The entry in the final column is the maximum number of parameters among Painlevé families with the corresponding leading exponents, after all constraints, including the Hamiltonian constraint, have been imposed. Note that the degree of freedom coming from translation of $s$ is included as a parameter (cf. Remark (3.11)). For $S=\{4\},\{6\},\{7\}$ or $\{8\}$ we obtain the largest number of parameters $(7)$ in the expansion by taking $n_{p}=0$. We get one fewer parameter if $n_{1}=1$.

(iii) Expansions with $N=1$ (i.e. expansions meromorphic in $s$ rather than a fractional power of $s$ ) are obtained except when $S$ is $\{1, \ldots, 8\},\{4,5\}$, or $\{1,2,3,6,7,8\}$.

Let us discuss the cases $S=\{1, \ldots, 8\}$ and $S=\{1,2,3,6,7,8\}$ in more detail.

Remark 5.4. AC Cases: $S=\{1, \ldots, 8\}$. In case (a) there are no nontrivial rational resonances, and the Painlevé expansion is trivial.

In case (b) we may choose $N$ to be 5 and obtain a Painlevé expansion depending on three parameters (from resonances at $-1, \frac{2}{5}, \frac{3}{5}$ ). The compatibility conditions either hold trivially or by Proposition (4.1).

Remark 5.5. ALC Case: $S=\{1,2,3,6,7,8\}$. The recursion and the compatibility conditions are non-trivial in this case and were worked out 
using MAPLE. Recall from the leading term analysis that we have a free parameter $\omega_{0}$, which corresponds to the resonance $R=0$. It also follows easily from $(1.6, i=4)$ that $n_{4}=-1 / 2$. The integer $N$ is chosen to be 4 , in view of the resonances.

At the resonance $R=1 / 4$ we have a free parameter $\omega_{1}$ given by

$$
\left(\beta_{1}^{(1)}, \beta_{1}^{(2)}, \beta_{1}^{(3)}\right)=\omega_{1}(1,-1,-\sqrt{2}) .
$$

At $R=\frac{1}{2}$ we encounter the first non-trivial compatibility condition $\omega_{0}+$ $4 \omega_{1}^{2}=0$ and a new free parameter $\omega_{2}=\beta_{2}^{(4)}$, as a result of (3.12) and $-n_{4}=\frac{1}{2}$. The compatibility condition at $R=\frac{3}{4}$ is identical to that at $R=\frac{1}{2}$. We also pick up a free parameter $\omega_{3}$ given by

$$
\left(\beta_{3}^{(1)}, \beta_{3}^{(2)}, \beta_{3}^{(3)}\right)=\omega_{3}(1,-1,-\sqrt{2}) .
$$

At $R=\frac{3}{2}$, we encounter the non-trivial compatibility condition

$$
\omega_{2}=\frac{1}{3 \sqrt{5}}\left(88192 \omega_{1}^{4}-2760 \omega_{1} \omega_{3}+2 \frac{\omega_{3}^{2}}{\omega_{1}^{2}}\right),
$$

and if it is satisfied, we pick up another free parameter $\omega_{4}$. By Proposition 4.1 and Remark 4.6 the compatibility condition at the top resonance $R=2$ is satisfied. The free parameter entering at this stage is used to satisfy the Hamiltonian constraint as indicated in (4.8). Therefore, we have a Painlevé expansion with 4 free parameters: $\omega_{1}, \omega_{3}, \omega_{4}$ and the position of the singularity.

The cohomogeneity 1 metrics with $G_{2}$-holonomy are given by the solutions of the system (1.5)-(1.8) which lie in the subvariety

$$
\begin{aligned}
v_{4} & = \pm \frac{\sqrt{5}}{2 \sqrt{2}}\left(\sqrt{-z_{4}}-\sqrt{-z_{5}}\right) \\
v_{3}+\frac{1}{\sqrt{5}} v_{4} & = \pm\left(-\sqrt{2} \sqrt{-z_{5}}-\sqrt{-z_{6}}-\sqrt{-z_{7}}+\sqrt{-z_{8}}\right) \\
\sqrt{2} v_{2}-v_{3}+\frac{1}{\sqrt{5}} v_{4} & = \pm 2\left(\sqrt{-z_{6}}-\sqrt{-z_{7}}-\sqrt{-z_{8}}\right) \\
\sqrt{2} v_{1}+\sqrt{2} v_{2}+v_{3}-\frac{1}{\sqrt{5}} v_{4} & = \pm\left(-\sqrt{2} \sqrt{-z_{4}}+\sqrt{-z_{6}}-\sqrt{-z_{7}}+\sqrt{-z_{8}}\right),
\end{aligned}
$$

where all square roots are positive and \pm depends on the orientation chosen. One can obtain these equations for example by transforming the $G_{2^{-}}$ equations in $[\mathrm{BGGG}$ ] to the variables we use. Hence the above subvariety 
can be parametrized by $x_{i}=z_{i} / A_{i}$ for $i=4,5,6,7,8$ together with the constraint $x_{4} x_{7}=x_{5} x_{8}$. One checks that, regardless of the \pm sign chosen above, the system (1.5)-(1.6) induces on the subvariety the system

$$
\zeta_{i}^{\prime}=\zeta_{i} \sum_{j} C_{i j} \zeta_{j}: \quad(i, j=4,5,6,7,8)
$$

where $\zeta_{i}$ is the positive square root of $x_{i}$ and $C$ is the matrix

$$
\left(\begin{array}{rrrrr}
\frac{-1}{\sqrt{2}} & 0 & 0 & -2 & 0 \\
0 & \frac{1}{\sqrt{2}} & 0 & 0 & -2 \\
0 & 0 & 1 & -2 & -2 \\
\frac{1}{\sqrt{2}} & 0 & -2 & 1 & -1 \\
0 & \frac{-1}{\sqrt{2}} & -2 & -1 & 1
\end{array}\right)
$$

By comparing the Painlevé expansions of this system with those of the full system having the same leading terms, we obtain

Theorem 5.6. Consider the cohomogeneity one Ricci-flat system for the principal orbit $\left((S U(2) \times S U(2) \times \Delta U(1)) \ltimes \mathbb{Z}_{2}\right) /\left(\Delta U(1) \times \mathbb{Z}_{2}\right) \approx S^{3} \times S^{3}$. The Painleve expansions of the associated quadratic system (1.5)-(1.8) which contain metrics of $G_{2}$-holonomy are given by

(I) a 3-parameter family of convergent Painlevé expansions representing asymptotically conical Ricci-flat ends, within which lies a 2-parameter subfamily representing $A C$ metrics with $G_{2}$-holonomy;

(II) a 4-parameter family of convergent Painlevé expansions representing asymptotically locally conical Ricci-flat ends, within which lie two 3parameter subfamilies representing $A L C$ metrics with $G_{2}$-holonomy;

(III) a 7-parameter family of convergent Painlevé expansions representing local Ricci-flat metrics, within which lies a 4-parameter subfamily representing metrics with $G_{2}$-holonomy.

Remark 5.7. The two parameters of the family of AC $G_{2}$-metrics come from resonances at -1 and $\frac{3}{5}$. These metrics were discovered in [BS] and [GPP].

The subsets of ALC $G_{2}$-metrics are given by the cubic equations $\omega_{3}=$ $32 \omega_{1}^{3}$ and $\omega_{3}=1328 \omega_{1}^{3}$, where $\omega_{1}, \omega_{3}, \omega_{4}$ are the parameters described in Remark (5.5). These $G_{2}$-metrics were first found in [BGGG]

The third family in the above theorem corresponds to the case $S=\{4\}$ with $n_{1}=0$. While the family depends on the full number of parameters, only one of the dependent variables actually blows up. The corresponding 
cohomogeneity one manifolds have orbit space a finite interval $\left(0, t_{0}\right)$, and near $t=0, f_{1}(t) \sim t^{-1 / 3}, f_{3}(t) \sim t^{1 / 3}$, and $f_{2}(t), f_{4}(t) \sim$ const.

We next discuss Painlevé expansions in which some $z_{i}$ has leading exponent less than -2 .

Using Remark (2.14) one sees after some computation that the only possibility for $\tilde{S}$ is $\{1,7,8\}$ and

$$
\left(\alpha_{0}^{(1)}, \ldots, \alpha_{0}^{(8)}\right)=\left(-2 a, b, b, c, c, \frac{b^{2}}{16 a}, a, a\right)
$$

where $b>0, a<0, c<0$ are free parameters. In writing down the recursion relations, (3.5) remains the same, but as we split the right-hand sum in (3.4) into two using the definition of $\tilde{S}$ in (2.14), the terms involving $m_{i} \in \tilde{S}$ actually occur later in the recursion. Let $\tilde{m}$ denote the minimum value of the leading exponents in the $z_{i}$. For $p=1,2,3$, we now rename the index $j-N\left(\tilde{m}-n_{p}+1\right)$ as $j$, and obtain

$$
\epsilon_{p} \sum_{i \in \tilde{S}} Q_{i p} \alpha_{j}^{(i)}=\left(\frac{j}{N}+\tilde{m}+1\right) \beta_{j+N\left(\tilde{m}-n_{p}+1\right)}^{(p)}-\epsilon_{p} \sum_{i \notin \tilde{S}} Q_{i p} \alpha_{j-N\left(m_{i}-\tilde{m}\right)}^{(i)} .
$$

As for $p=4$, since $Q_{i 4}=0$ unless $i=4,5,(3.4)$ can be written as

$$
-\left(\frac{j}{N}+n_{4}\right) \beta_{j}^{(4)}+\epsilon_{4} \sum_{i=4,5} Q_{i 4} \alpha_{j-N\left(m_{i}-n_{4}+1\right)}^{(i)}=0 .
$$

Note that either $n_{4}-1=m_{4}=m_{5}$, or else $n_{4}=0$ (which can happen only when $4 \notin T)$.

In the former case, (3.4) in matrix form becomes

$$
\Gamma \alpha_{j}-\operatorname{diag}\left(0,0,0, n_{4}+(j / N)\right) \beta_{j}=\tilde{Y}_{j},
$$

where

$$
\Gamma=\left(\begin{array}{rrrrrrrr}
\sqrt{2} & 0 & 0 & 0 & 0 & 0 & \frac{3}{\sqrt{2}} & \frac{1}{\sqrt{2}} \\
-\sqrt{2} & 0 & 0 & 0 & 0 & 0 & -\frac{3}{\sqrt{2}} & -\frac{1}{\sqrt{2}} \\
0 & 0 & 0 & 0 & 0 & 0 & -1 & 1 \\
0 & 0 & 0 & -\frac{\sqrt{5}}{2} & -\frac{\sqrt{5}}{2} & 0 & 0 & 0
\end{array}\right)
$$

and

$$
\tilde{Y}_{j}^{(p)}= \begin{cases}\left(\frac{j}{N}+\tilde{m}+1\right) \beta_{j+N\left(\tilde{m}-n_{p}+1\right)}^{(p)}-\epsilon_{p} \sum_{i \notin S} Q_{i p} \alpha_{j-N\left(m_{i}-\tilde{m}\right)}^{(i)} & \text { if } p=1,2,3 \\ 0 & \text { if } p=4 .\end{cases}
$$


Eliminating $\alpha_{j}$ in (5.9) using (3.5), we obtain the following analogue of (3.7):

$$
\begin{aligned}
& \left(2 \Gamma \operatorname{diag}\left(\alpha_{0}^{(1)}, \ldots, \alpha_{0}^{(8)}\right) Q_{T}-(j / N) \operatorname{diag}\left(0,0,0, n_{4}+(j / N)\right)\right) \beta_{j} \\
& =\left(\frac{j}{N}\right) \tilde{Y}_{j}-\Gamma X_{j},
\end{aligned}
$$

where $Q_{T}$ is the matrix obtained from $Q$ by replacing each column whose index does not lie in $T$ by a corresponding column of zeros.

Let $\tilde{M}_{j}$ denote the matrix on the left of $\beta_{j}$ in (5.10). The new feature here is that for all $j \geq 1$ the matrix $\tilde{M}_{j}$ has rank 2 . Indeed its left kernel is spanned by the vectors $(1,1,0,0)$ and $(\sqrt{2}, 0,1,0)$, and so the compatibility condition at step $j$ is that the right side of (5.10) should be annihilated by these vectors.

We first analyse the compatibility conditions for $1 \leq j \leq(-2-\tilde{m}) N$. As a result we are able to conclude that all $m_{i} \geq-2$ whenever $i \notin \tilde{S}$, and the only subsets $T$ for which all these compatibility conditions hold are (i) $T=\{1,2,3\}$ and (ii) $T=\{1,2,3,4\}$.

If $n_{4}=0$ instead, we arrive at the same conclusions except that only possibility (i) for $T$ can arise. However, the arguments require some minor modifications. For example, the last row of the matrix $\Gamma$ becomes zero, and $Y_{j}^{(4)}$ is the negative of the second term on the left in (5.8).

For case (i), we find that the vector of leading exponents is

$$
(-8 / 3,-2,-2,-4 / 3,-4 / 3,-4 / 3,-8 / 3,-8 / 3),
$$

and in the vector of leading coefficients $b=2 / 3$. So $\tilde{m}=-8 / 3,-2-\tilde{m}=2 / 3$, and $n_{4}=-1 / 3$. (The case $n_{4}=0$ leads to a contradiction.) One can show that the two free parameters introduced at step $j$ are fixed by the compatibility condition at step $j+(2 N / 3)$ as long as $j \geq 1$ is not equal to $N / 3, N$ or $2 N$. Hence it is natural to choose $N=3$ in our expansion. Using MAPLE to perform the recursion up to step $j=8$, we obtain a Painlevé family depending on 5 parameters, satisfying all the constraints, including the Hamiltonian constraint.

Regarding the asymptotics of this family, one readily checks that it represents complete Ricci flat ends and the quantity $\sum_{j} U^{i j} m_{j}=0$ for precisely $i=1,2$. Also, $f_{3}(t), f_{4}(t) \sim t$. So the Ricci-flat ends are asymptotic to a 2-torus bundle over a cone on $S^{2} \times S^{2}$. We can therefore state

Theorem 5.11. For the quadratic system in Theorem (5.6), there is also a 5 -parameter family of convergent Painlevé expansions representing complete Ricci-flat ends asymptotic to a 2-torus bundle over a cone on $S^{2} \times S^{2}$. 
For case (ii), we find that the vector of leading exponents is

$$
(2(b-2),-2,-2,-2,-2,-2 b, 2(b-2), 2(b-2)),
$$

where $b$ is the same parameter occurring in the vector of leading coefficients. We further have $c=1-\left(\frac{3}{2}\right) b$ and $\frac{2}{3}<b<1$. Accordingly, let $b=\frac{p}{N}$ where $p<N$ are relatively prime. For a generic choice of $b$ (meaning that the equations $R^{2}-R+5-\left(\frac{15}{2}\right) b=0$ and $2 R^{4}-4 R^{3}+(8-15 b) R^{2}-(6-15 b) R-$ $30 b\left(1-\left(\frac{3}{2}\right) b\right)=0$ have no positive rational roots), one can show that the two free parameters introduced at step $j \geq 1$ are fixed by the compatibility condition at step $j+2 N-2 p$ except possibly when $j=N$. So we would get a Painlevé expansion depending on at most 2 parameters, satisfying all constraints. Among non-generic values for $b$, the most interesting one is $b=\frac{3}{4}$ because in this case $f_{2}(t)$ would be asymptotically constant. However, the compatibility condition at $j=6$ cannot be satisfied.

One can check that there are no exceptional holonomy metrics in the Painlevé expansions with leading exponents which are less than -2 .

\section{6. $S^{4 k+3}$ as principal orbit and $\operatorname{Spin}(7)$ metrics.}

Example 6.1. Principal orbit $S^{4 k+3} \approx G / K=S p(k+1) U(1) / S p(k) \Delta(U(1))$.

In this orbit, the $S p(k+1)$ acts via left multiplication on $S^{4 k+3}$ and the $U(1)$ acts by right multiplication. We have inclusions

$\mathfrak{s p}(k) \oplus \Delta \mathfrak{u}(1) \hookrightarrow \mathfrak{s p}(k) \oplus(\mathfrak{u}(1) \oplus \mathfrak{u}(1)) \hookrightarrow \mathfrak{s p}(k) \oplus \mathfrak{s p}(1) \oplus \mathfrak{u}(1) \hookrightarrow \mathfrak{s p}(k+1) \oplus \mathfrak{u}(1)$

whose successive quotients give the decomposition of the isotropy representation $\mathfrak{p}$ into the sum of three irreducible summands, of dimension 1,2 and $4 k$. (The first, $\mathfrak{p}_{1}$, is a trivial summand, $\mathfrak{p}_{2}$ is an irreducible representation of $U(1)$, and $\mathfrak{p}_{3}$ is a copy of $\mathbb{H}^{k}$.) The scalar curvature function involves weight vectors $w^{(1)}, \ldots, w^{(5)}$ given respectively by

$$
(0,-1,0),(0,0,-1),(1,-2,0),(1,0,-2),(0,1,-2),
$$

and the constants $A_{i}$ are

$$
\left(A_{1}, \ldots, A_{5}\right)=\left(4,4 k(k+2),-\frac{1}{2},-\frac{k}{4},-k\right),
$$

where the background metric $B$ is the direct sum of the trace forms $-\operatorname{tr}(X Y)$ on $S U(2 k+2)$ and $S U(2)$. 
We therefore have $r=3$ and $\ell=2$. The matrix $\Phi$ is given by

$$
\Phi=\left(\begin{array}{ccccc}
\frac{1}{2} & 1 & 0 & 1 & \frac{3}{2} \\
1 & \frac{4 k-1}{4 k} & 1 & \frac{2 k-1}{2 k} & \frac{2 k-1}{2 k} \\
0 & 1 & -2 & 0 & 2 \\
1 & \frac{2 k-1}{2 k} & 0 & -\frac{1}{k} & \frac{k-1}{k} \\
\frac{3}{2} & \frac{2 k-1}{2 k} & 2 & \frac{k-1}{k} & \frac{k-2}{2 k}
\end{array}\right)
$$

and a choice of $Q$ is

$$
Q=\left(\begin{array}{ccc}
\frac{1}{\sqrt{2}} & 0 & 0 \\
\sqrt{2} & \sqrt{\frac{4 k+1}{4 k}} & 0 \\
0 & -\sqrt{\frac{4 k}{4 k+1}} & \sqrt{\frac{4 k+2}{4 k+1}} \\
\sqrt{2} & \frac{2 k+1}{\sqrt{k(4 k+1)}} & \sqrt{\frac{4 k+2}{4 k+1}} \\
\frac{3}{\sqrt{2}} & \sqrt{\frac{4 k+1}{k}} & 0
\end{array}\right)
$$

It is well-known from [Z] that (up to homothety) there are only two $G$ invariant Einstein metrics on $G / K$. These are the round metric and the Jensen metric $[\mathrm{J}]$.

An analysis of the possible leading terms for the Painlevé expansions is summarised below. Note that we do not get any leading exponents which are less than -2 in this case.

\begin{tabular}{|c|c|c|c|c|}
\hline $\mathrm{S}$ & $\mathrm{T}$ & $\mathrm{m}$ & $\alpha_{0}^{(i)}(i \in S)$ & Remarks \\
\hline$\{4\}$ & $\{1,2,3\}$ & $(2 k, 2 k-1,0,-2,2 k-2)$ & $-k$ & \\
$\{3,4\}$ & $\{1,2,3\}$ & $(2 k, 2 k,-2,-2,2 k)$ & $-\frac{1}{2},-k$ & \\
$\{4,5\}$ & $\{1,2,3\}$ & $(8,3,8-2,-2)$ & $-1,-2$ & $k=1$ only \\
$\{1, \ldots, 5\}$ & $\{1,2,3\}$ & $(-2, \ldots,-2)$ & see $(6.2)$ & \\
$\{3\}$ & $\{2,3\}$ & $(0,1,-2,0,2)$ & $-\frac{1}{2}$ & \\
$\{5\}$ & $\{1,2\}$ & $(6,2,8,0,-2)$ & -2 & $k=1$ only \\
$\{1,2,5\}$ & $\{1,2\}$ & $\left(-2,-2,-\frac{8 k}{4 k+1},-\frac{8 k}{4 k+1},-2\right)$ & see $(6.2)$ & \\
\hline
\end{tabular}

Remark 6.2. The constraints (4.7) will impose further conditions on $\alpha_{0}$. In the case when $S=\{1, \ldots, 5\}$, there are two possibilities for $\alpha_{0}$ compatible with the constraints:

(a) $\alpha_{0}=\frac{1}{(2 k+1)^{2}}\left(2,4 k(k+2),-\frac{1}{2},-k,-2 k\right)$,

(b) $\alpha_{0}=\frac{(2 k+3)^{2}}{16 k^{3}+64 k^{2}+64 k+18}\left(4, \frac{8 k(k+2)}{2 k+3},-1,-\frac{2 k}{(2 k+3)^{2}},-\frac{4 k}{(2 k+3)^{2}}\right)$, corresponding to the round and Jensen metrics on the principal orbit respectively. 
If $S=\{1,2,5\}$ then the constraints force $\alpha_{0}$ to be given by or

(a) $\alpha_{0}=\frac{1}{(k+1)(4 k+1)}\left(2,4 k(k+2), \mu_{0}, 2 k \mu_{0},-2 k\right)$,

(b) $\alpha_{0}=\frac{1}{(4 k+1)\left(k^{2}+3 k+1\right)}\left(2(k+1)^{2}, 4 k(k+1)(k+2), \mu_{0}, \frac{2 k}{(k+1)^{2}} \mu_{0},-2 k\right)$, where $\mu_{0}<0$ is a free parameter.

The quantity $1-\frac{1}{2} \sum_{j} m_{j} \xi_{j}$ is negative when $S=\{1,2,3,4,5\}$ or $\{1,2,5\}$, and is positive in the remaining cases.

If $S=\{1, \ldots, 5\}$ we have AC asymptotics. When $S=\{1,2,5\}$, the quantity $\sum_{j} U^{i j} m_{j}$ is 0 precisely for $i=1$. Since the summand $\mathfrak{p}_{1}$ is trivial, and we can check that $f_{2}(t), f_{3}(t)$ grow linearly, we have complete Ricci-flat ends with ALC asymptotics in this case.

In the cases where $1-\frac{1}{2} \sum_{j} m_{j} \xi_{j}>0$, the volume of the principal orbits tends to 0 as $s$ tends to 0 , but there is always at least one $f_{i}(t)^{2}$ which blows up. In the cases of $S=\{3\},\{4\},\{5\}$, there is a unique $i$ such that $f_{i}(t)^{2}$ is asymptotically a positive constant.

We list next the results of the analysis of the resonances and compatibility conditions. We follow the same conventions in Remark (5.3).

\begin{tabular}{|c|c|c|}
\hline $\mathrm{S}$ & Resonances (multiplicities) & $\begin{array}{c}\text { number of } \\
\text { parameters } \\
\text { in expansion }\end{array}$ \\
\hline$\{4\}$ & $-1,0$ (twice), 1 (twice), 2 & 5 \\
$\{3,4\}$ & -1 (twice), $0,1,2$ (twice) & 4 \\
$\{4,5\}$ & -1 (twice), $0,1,2$ (twice) & 4 \\
$\{1, \ldots, 5\}$ (a) & $-1,-\frac{1}{2 k+1}($ twice $) \frac{2 k+2}{2 k+1}($ twice $), 2$ & 3 \\
$\{1, \ldots, 5\}$ (b) & $-1,2$, roots of $R(R-1)=\frac{2(k+1)(4 k+9)}{(2 k+1)\left(4 k^{2}+14 k+9\right)}$, & 4 if $k=1$ \\
& roots of $R(R-1)=-\frac{\left(4 k^{2}+10 k+6\right)}{(2 k+1)\left(4 k^{2}+14 k+9\right)}$ & 1 if $k>1$ \\
$\{3\}$ & $-1,0$ (twice) 1,2 and 0 or $-n_{1}$ & 5 \\
$\{5\}$ & $-1,0($ twice) $, 1,2$ and 0 or -1 & 5 \\
$\{1,2,5\}$ (a) & $-1,0,2$, roots of $R(R-1)=\frac{2 k}{(k+1)(4 k+1)}$ and $\frac{4 k-1}{4 k+1}$ & 3 \\
$\{1,2,5\}$ (b) & $-1,0,2$ roots of $R(R-1)=-\frac{2 k(k+1)}{(4 k+1)\left(k^{2}+3 k+1\right)}$ and $\frac{4 k-1}{4 k+1}$ & 4 if $k=1$ \\
& & 3 if $k>1$ \\
\hline
\end{tabular}

Note that we get expansions with $N=1$ except when $S=\{1, \ldots, 5\}$ or $\{1,2,5\}$. Also, when $S=\{3\}, n_{1}$ is either 0 or $\geq 1$, and the largest number of parameters occurs when $n_{1}=0$.

Let us discuss the cases when $S=\{1, \ldots, 5\}$ or $S=\{1,2,5\}$ in more detail. 
AC Cases: $S=\{1, \ldots, 5\}$.

(a) The eigenvalues of the matrix $M$ are 2 and $\frac{2 k+2}{(2 k+1)^{2}}$, the latter with multiplicity 2 . Hence the resonances are as given in the above table. The compatibility condition at the first positive resonance is trivial, while that at the top resonance is handled by (4.1). It follows that we obtain a 3-parameter Painlevé expansion for all $k \geq 1$.

(b) The equation $R(R-1)=-\frac{4 k^{2}+10 k+6}{(2 k+1)\left(4 k^{2}+14 k+9\right)}$ has rational roots iff

$$
\left(8 k^{3}+16 k^{2}-8 k-15\right)(2 k+1)\left(4 k^{2}+14 k+9\right) \text { is a perfect square. }
$$

This condition arose already in Example 6.4 of [DW4]. It is certainly satisfied when $k=1$. In $\S 7$ we will show that there are only finitely many solutions of (6.3) and provide strong evidence that $k=1$ is the only positive integer solution.

The equation $R(R-1)=\frac{2(k+1)(4 k+9)}{(2 k+1)\left(4 k^{2}+14 k+9\right)}$ has rational roots iff

$$
\left(8 k^{3}+64 k^{2}+136 k+81\right)(2 k+1)\left(4 k^{2}+14 k+9\right) \text { is a perfect square. }
$$

This condition is satisfied for $k=1$ and for at most finitely many positive integers. We again conjecture that $k=1$ is the only positive integer solution for (6.4), and provide strong evidence for our conjecture in $\S 7$.

Therefore, if $k>1$ we do not expect any nontrivial Painlevé expansions in this case.

If $k=1$, on the other hand, we obtain resonances at $-1,-\frac{4}{9}, \frac{4}{9}, \frac{5}{9}, \frac{13}{9}$ and 2. The compatibility conditions at $R=\frac{4}{9}, \frac{5}{9}$ hold trivially and at the top resonance $R=2$ we may use Proposition (4.1). So the only non-trivial compatibility condition is at $R=\frac{13}{9}$, which we can show holds using MAPLE. Hence, we obtain a 4-parameter Painlevé expansion in powers of $s^{1 / 9}$. These metrics are asymptotic to the cone over the Jensen sphere.

ALC Cases: $S=\{1,2,5\}$.

The conditions for rationality of the resonances arising from eigenvalues of $M_{T}$ are identical to those analysed in Example 6.2 of [DW4] for a Ricciflat $(4 k+3)$-manifold with principal orbit $\mathbb{C P}^{2 k+1}=S p(k+1) / S p(k) U(1)$. This can be attributed to the fact that the Ricci-flat metric in our present paper asymptotically approaches a circle bundle of constant length over a cone over $S p(k+1) / S p(k) U(1)=\mathbb{C P}^{2 k+1}$. It follows from the analysis of [DW4] that case (a) never gives nontrivial rational resonances besides $\frac{4 k-1}{4 k+1}$. We obtain a 3-parameter family of Painlevé expansions. The compatibility 
condition at $\frac{4 k-1}{4 k+1}$ follows easily since it is the first nonzero resonance, and the compatibility condition at the top resonance follows from applying part (v) of Proposition 4.1.

In case (b), recall that the rationality condition is that

$$
\left(4 k^{3}+5 k^{2}-k+1\right)\left(k^{2}+3 k+1\right)(4 k+1) \text { is a perfect square. }
$$

This condition is satisfied for $k=1$ and for at most finitely many $k$. Again we conjecture that this is the only positive integer solution for (6.5) and will provide strong evidence for our conjecture in $\S 7$.

We will therefore focus on case (b) with $k=1$. Note that from (1.6) with $i=3$ we must have $n_{3}=\frac{3}{5}$. Hence we may choose the integer $N$ to be 5 since the resonances are $-1,0, \frac{1}{5}, \frac{3}{5}, \frac{4}{5}$ and 2 . We then use MAPLE to check the compatibility conditions. Recall from (6.2) that the leading coefficients contain a free parameter $\mu_{0}$. A free parameter $\mu_{1}$ enters at $R=\frac{1}{5}$ from the relation $\left(\beta_{1}^{(1)}, \beta_{1}^{(2)}\right)=\mu_{1}\left(1, \frac{-\sqrt{10}}{2}\right)$. At $R=\frac{3}{5}$, we get a non-trivial compatibility condition from (3.12): either $\mu_{1}=0$ or $225 \mu_{1}^{2}+\mu_{0}=0$. If this is satisfied, then $\beta_{3}^{(3)}$ becomes a free parameter $\mu_{2}$. At $R=\frac{4}{5}$ the compatibility condition is

$$
\left(225 \mu_{1}^{2}+\mu_{0}\right)\left(1125 \mu_{1}^{2}+\mu_{0}\right)=0,
$$

which holds iff the earlier compatibility condition $225 \mu_{1}^{2}+\mu_{0}=0$ holds, and then we get another free parameter $\mu_{3}$. Finally, by Proposition 4.1 and Remark 4.6 the compatibility condition holds at the top resonance, but the free parameter that enters at that stage is needed to fix the Hamiltonian constraint. So we obtain a Painlevé expansion with 4 parameters: $\mu_{1}, \mu_{2}, \mu_{3}$ and the position of the singularity.

The situation when $k>1$ is similar to that of case (a).

The cohomogeneity one metrics with $\operatorname{Spin}(7)$-holonomy are given by the solution curves of the Ricci-flat system (1.5)-(1.8) which lie on the subvariety defined by

$$
\begin{aligned}
\sqrt{30} v_{3} & = \pm 3\left(\sqrt{x_{3}}-\sqrt{x_{4}}\right) \\
-\sqrt{3} v_{2}-\sqrt{2} v_{3} & = \pm \sqrt{15}\left(\frac{1}{2} \sqrt{x_{4}}+\sqrt{2} \sqrt{x_{5}}\right) \\
3 \sqrt{5} v_{1}+4 \sqrt{2} v_{2}-\sqrt{3} v_{3} & = \pm 3 \sqrt{5}\left(2 \sqrt{x_{1}}-\frac{1}{\sqrt{2}} \sqrt{x_{3}}-\sqrt{x_{5}}\right),
\end{aligned}
$$

where $x_{i}=z_{i} / A_{i}$, all square roots are positive, and \pm depends on the chosen orientation. These equations can be deduced for example from the $\operatorname{Spin}(7)$ equations in [CGLP1] by a change of variables to ours. Hence the above 
subvariety can be parametrised by $\zeta_{i}=\sqrt{x_{i}}$ for $i=1,3,4,5$ together with the constraint $x_{1} x_{4}=x_{3} x_{5}$. The Hamiltonian vector field is tangent to this subvariety and induces on it the system

$$
\zeta_{i}^{\prime}=\zeta_{i} \sum_{j} \tilde{C}_{i j} \zeta_{j}: \quad(i, j:=1,3,4,5)
$$

where $\tilde{C}$ is the matrix

$$
\left(\begin{array}{rrrr}
-\sqrt{2} & 0 & -\frac{1}{2} & \frac{-3}{\sqrt{2}} \\
0 & -1 & 0 & -2 \sqrt{2} \\
-2 \sqrt{2} & 0 & \frac{1}{2} & 0 \\
-3 \sqrt{2} & 1 & 0 & \frac{1}{\sqrt{2}}
\end{array}\right)
$$

By comparing the Painlevé expansions of this system which have the same leading terms as those of the full Ricci-flat system, we obtain

Theorem 6.6. Consider the cohomogeneity one Ricci-flat system for the principal orbit $G / K=(S p(k+1) \cdot U(1)) /(S p(k) \cdot \Delta U(1)) \approx S^{4 k+3}$. For any $k \geq 1$, the associated quadratic system (1.5)-(1.8) has

(i) a 3-parameter family of convergent Painlevé expansions representing complete Ricci-flat ends asymptotic to the metric cone over the round metric on $S^{4 k+3}$,

(ii) a 3-parameter family of convergent Painlevé expansions representing complete Ricci-flat ends asymptotic to a circle bundle over a cone on $\mathbb{C P}^{2 k+1}$.

These Painlevé families have generic holonomy.

When $k=1$, i.e., when $G / K \approx S^{7}$, the quadratic system has in addition

(I) a 4-parameter family of convergent Painlevé expansions representing complete Ricci-flat ends asymptotic to the cone over the Jensen metric on $S^{7}$, within which lies a 2-parameter family of Painlevé expansions representing metrics with $\operatorname{Spin}(7)$-holonomy,

(II) a 4-parameter family of convergent Painlevé expansions representing complete Ricci-flat ends with ALC asymptotics, within which lies a 3parameter family of Painlevé expansions representing metrics with Spin(7)holonomy.

(III) a 5-parameter family of meromorphic Painlevé expansions representing local Ricci-flat metrics, within which lies a 3-parameter subfamily representing metrics with Spin(7)-holonomy.

(I)-(III) are the only Painlevé families which contain metrics with $\operatorname{Spin}(7)$-holonomy. 
Remark 6.7. Our conjecture $(\operatorname{cf} \S 7)$ about the solutions of the Diophantine problems (6.3)-(6.5) implies that the 4-parameter families (I) and (II) are unique to the $k=1$ case.

In (I), the resonances of the $\operatorname{Spin}(7)$-subsystem are $-1,-\frac{4}{9}, 0, \frac{5}{9}$. The free parameter at $R=0$ is fixed by the constraint $x_{1} x_{4}=x_{3} x_{5}$, leaving the free parameter at $R=\frac{5}{9}$ and the position of the singularity. These AC $\operatorname{Spin}(7)$-metrics were found in [BS] and [GPP].

In (II), the resonances of the $\operatorname{Spin}(7)$-subsystem are $-1,0,0, \frac{4}{5}$, and the constraint $x_{1} x_{4}=x_{3} x_{5}$ fixes one of the free parameters at $R=0$. In terms of the parameters $\mu_{1}, \mu_{2}, \mu_{3}$ in the Ricci-flat Painlevé expansions, the $\operatorname{Spin}(7)$ metrics are cut out by the equation

$$
\sqrt{\frac{5}{3}} \mu_{2}=-\frac{45}{4 \sqrt{2}} \mu_{1} \mu_{3}+\frac{2179 \cdot 5^{4} \cdot 9}{49 \cdot 16} \mu_{1}^{5} .
$$

Note that in this example we have a Painlevé expansion for the $\operatorname{Spin}(7)$ subsystem depending on the full number of parameters (three), in which all the dependent variables blow up. This is consistent with the fact that these $\operatorname{Spin}(7)$-equations are an integrable system-they are solved in [CGLP1] in terms of hypergeometric functions.

The expansions in (III) correspond to the case $S=\{3\}$ with $n_{1}=0$. The cohomogeneity one Ricci-flat manifolds have a finite interval $\left(0, t_{0}\right)$ as orbit space. Near $t=0, f_{1}(t) \sim t^{-1 / 3}, f_{2}(t) \sim t^{1 / 3}$, and $f_{3}(t) \sim$ const.

Remark 6.8. It is interesting to compare the example analysed in this section with the case, analysed in [DW4], when the principal orbit $S^{4 k+3}$ is viewed instead as the homogeneous space $(S p(k+1) S p(1)) /(S p(k) \Delta S p(1))$. In the latter case there are only two irreducible summands in the isotropy representation, and not all $S p(k+1) U(1)$-invariant metrics on $S^{4 k+3}$ are $S p(k+1) S p(1)$-invariant.

This means that the Ricci-flat equations become a subsystem of the system considered above. In particular the expansions for the subsystem will have four rather than six resonances.

We still have two possibilities for the cone-type leading terms, corresponding to the round and Jensen metrics, since these metrics are in fact $S p(k+1) S p(1)$-invariant [J], [Z]. The resonances in the two situations are now respectively

(a) $-1,-\frac{1}{2 k+1}, \frac{2 k+2}{2 k+1}, 2$, and

(b) $-1,2$ and the roots of $R(R-1)=-\frac{4 k^{2}+10 k+6}{(2 k+1)\left(4 k^{2}+14 k+9\right)}$.

It was shown in [DW4] that (a) gives a 2-parameter family of Painlevé expansions to the Ricci-flat equations. If $k=1$, (b) gives a 3-parameter AC 
Ricci-flat family (which includes the 2-parameter subfamily of AC Spin(7)metrics of [BS], [GPP] mentioned above), while if $k>1$, our conjecture would imply that we only get a trivial Painlevé expansion. Note that we do not get any expansions of ALC type.

Remark 6.9. We observe that if the choice of principal orbit type permits ALC asymptotics (as is the case in Examples 5.2 and 6.1, but not Example 5.1 or Remark 6.8), then the ALC Painlevé expansions for the exceptional holonomy subsystem involve more free parameters than the expansions representing $\mathrm{AC}$ metrics. This is in accord with the behaviour noticed by Cvetič-Gibbons-Lü-Pope, that ALC asymptotics seem to be "preferred" in some sense.

\section{Remarks on the Diophantine conditions.}

We will end by explaining the evidence for our conjecture that the only positive integer solution of the Diophantine problems (6.3), (6.4) and (6.5) is $k=1$.

First, by elementary arguments (cf [DW4]) it follows that (6.3), (6.4), (6.5) imply respectively that the following must be squares:

(a) $8 k^{3}+16 k^{2}-8 k-15,(2 k+1) / 3,\left(4 k^{3}+14 k+9\right) / 3$

(b) $8 k^{3}+64 k^{2}+136 k+81,(2 k+1) / 3,\left(4 k^{2}+14 k+9\right) / 3$

(c) $4 k^{3}+5 k^{2}-k+1,(4 k+1) / 5,\left(k^{2}+3 k+1\right) / 5$.

In particular, this shows that solutions of our Diophantine problems give integral points $(x, y)$ on the elliptic curves

$$
\begin{aligned}
& y^{2}=x^{3}+4 x^{2}-4 x-15, \quad(x=2 k) \\
& y^{2}=x^{3}+16 x^{2}+68 x+81, \quad(x=2 k) \\
& y^{2}=x^{3}+5 x^{2}-4 x+16, \quad(x=4 k)
\end{aligned}
$$

respectively, where $x$ is positive and has the divisibility property shown. Observe that for each such integral point we still have to check that the remaining linear and quadratic expressions are squares.

We summarise our findings below.

(i) A classical theorem of Siegel tells us there are only finitely many integral points on the elliptic curves, so each of our Diophantine problems has only finitely many solutions. 
(ii) We applied the program RATPOINTS of Elkies, Stahlke and Stoll (which finds rational points with given bounds on numerator and denominator on curves of the form $\left.y^{2}=p(x)\right)$ to our Diophantine equations. We found that in each case $k=1$ is the only integer solution in the range $1 \leq k \leq 10^{6}$.

(iii) We also used the program SIMATH to find all integral points on the elliptic curves (7.1)-(7.3). Using these integral points we again found that for each of our Diophantine problems $k=1$ is the only positive integral solution.

However, the algorithm for finding all integral points on a given elliptic curve uses first an algorithm to determine a basis for the free part of the Mordell-Weil group of the curve. As far as we understand, this last algorithm may, depending on the given curve, assume the conjecture of Birch/Swinnerton-Dyer. According to SIMATH, the Mordell-Weil groups of the elliptic curves (7.1)-(7.3) are torsion free and have respectively rank 2,3 and 2. We refer the interested reader to the webpage diana.math.unisb.de/ simath for further information.

Finally, the linear and quadratic parts of our Diophantine problems can also be used to show that $k=1$ is the only solution in the range $1 \leq$ $k \leq N$ for large $N$. This approach is more elementary since it uses only the classical methods for determining elements of a fixed norm in a real quadratic extension of the rationals. For example, in case (c) the relevant extension is $\mathbb{Q}(\sqrt{5})$. But it appears that one needs to involve the elliptic curves in order to prove that the number of solutions is finite.

\section{References.}

[ARS] M. Ablowitz, A. Ramani and H. Segur: A connection between nonlinear evolution equations and ordinary differential equations of P-type I, J. Math. Phys., 21, 715-721, (1980); II. ibid. 21, 1006-1015, (1980).

[AvM] M. Adler and P. van Moerbeke: Kowalewski's asymptotic method, Kac-Moody Lie algebras and regularization, Comm. Math. Phys., 83, 83-106, (1982)

[BGPP] V. Belinskii, G.W. Gibbons, D.N. Page and C.N. Pope: Asymptotically Euclidean Bianchi IX metrics in quantum gravity, Phys. Lett. B., 76, 433-435, (1978).

[BB] L. Bérard Bergery: Sur de nouvelles variétés riemanniennes d'Einstein, L'Institut Élie Cartan, Nancy (1982). 
[Bes] A. Besse: Einstein Manifolds, Ergebnisse der Math. und ihrer Grenzbegiete, 3. Folge, Band 10, Springer-Verlag, (1987).

[BWZ] C. Böhm, M. Wang and W. Ziller: A variational approach for compact homogeneous Einstein manifolds, Preprint 2003.

[BGGG] A. Brandhuber, J. Gomis, S. Gubser and S. Gukov: Gauge theory at large $N$ and new $G_{2}$ holonomy metrics, arXiv: hep-th/0106034, Nucl. Phys. B., 611, 179-204 (2001).

[B] R. Bryant: Metrics with exceptional holonomy, Ann. Math., 126, 525-576, (1987).

[BS] R. Bryant and S.M. Salamon: On the construction of some complete metrics with exceptional holonomy, Duke Math J., 58, 829850, (1989).

[CS] R. Cleyton and A.F. Swann: Cohomogeneity one $G_{2}$-structures, J. Geom. Phys., 44, 202-220, (2002).

[CGLP1] M. Cvetič, H. Lü, G.W. Gibbons and C.N. Pope: New complete non-compact Spin(7) manifolds, arXiv: hep-th/0103155, Nucl. Phys. B., 620, 29-54, (2002).

[CGLP2] M. Cvetič, H. Lü, G.W. Gibbons and C.N. Pope: Cohomogeneity one manifolds of $\operatorname{Spin}(7)$ and $G_{2}$ holonomy, arXiv: hepth/0108245, Ann. Phys., 300, 139-184, (2002).

[DW1] A. Dancer and M. Wang: The cohomogeneity one Einstein equations from the Hamiltonian viewpoint, J. reine angew. Math, 524, 97-128, (2000).

[DW2] A. Dancer and M. Wang: The cohomogeneity one Einstein equations and Painlevé Analysis, J. Geom. Phys., 38, 183-206, (2001).

[DW3] A. Dancer and M. Wang: Ricci-flat warped products and Painlevé analysis, J. Math. Phys., 42, 3609-3614, (2001).

[DW4] A. Dancer and M. Wang: Painlevé expansions and the Einstein equations:the two-summand case, J. Geom. Phys., 48, 12-43, (2003).

[DW5] A. Dancer and M. Wang: Integrability and the Einstein equations, in Symplectic and contact topology, (eds. Y. Eliashberg, B. Khesin 
and F. Lalonde), Fields Institute Communications, Vol. 35, 89$101,(2003)$.

[DW6] A. Dancer and M. Wang: Painlevé analysis of the Ricci-flat ordinary differential equations associated with Aloff-Wallach spaces and $U(1)$-bundles over Fano products, J. Math. Phys., 44, 3383$3406,(2003)$.

[EW] J. Eschenburg and M. Wang: The initial value problem for cohomogeneity one Einstein metrics, J. Geom. Anal., 10, 109-137, (2000).

[GPP $] \quad$ G.W. Gibbons, D. Page and C.N. Pope: Einstein metrics on $\mathbb{R}^{3}$, $\mathbb{R}^{4}$ and $S^{3}$ bundles, Comm. Math. Phys., 127, 529-553, (1990).

[J] G. Jensen: Einstein metrics on principal fibre bundles, J. Diff. Geom., 8, 599-614, (1973).

[K] S. Kowalewski: Sur le probleme de la rotation d'un corps solide autour d'un point fixe, Acta Math., 12, 177-232, (1889).

[St] M. Struwe: Variational methods. Applications to nonlinear partial differential equations and Hamiltonian systems, 2nd edition, Springer-Verlag, (1996).

[WZ] M. Wang and W. Ziller: On normal homogeneous Einstein manifolds, Ann. scient. Éc. Norm. Sup., $4^{e}$ série, t. 18, 563-633, (1985).

[Z] W. Ziller: Homogeneous Einstein metrics on spheres and projective spaces, Math. Ann., 259, 351-358, (1982).

ANDREW DANCER

Jesus College, Oxford University

OXFORD, OX1 3DW

UNITED KINGDOM

dancer@maths.ox.ac.uk

MCKenZie Y. WANG

Department of Mathematics and Statistics, McMaster University

Hamilton, OnTARIO, L8S 4K1

Canada

wang@mcmaster.ca

Received August 8, 2002. 